Maik Liebl*, Dietmar Eberbeck, Annelies Coene, Jonathan Leliaert, Philine Jauch, Margarita Kruteva, Lisa Fruhner, Lester Barnsley, Stefan G. Mayr and Frank Wiekhorst

\title{
9 Magnetic measurement methods to probe nanoparticle-matrix interactions
}

\begin{abstract}
Magnetic nanoparticles (MNPs) are key elements in several biomedical applications, e.g., in cancer therapy. Here, the MNPs are remotely manipulated by magnetic fields from outside the body to deliver drugs or generate heat in tumor tissue. The efficiency and success of these approaches strongly depend on the spatial distribution and quantity of MNPs inside a body and interactions of the particles with the biological matrix. These include dynamic processes of the MNPs in the organism such as binding kinetics, cellular uptake, passage through cell barriers, heat induction and flow. While magnetic measurement methods have been applied so far to resolve the location and quantity of MNPs for therapy monitoring, these methods can be advanced to additionally access these particle-matrix interactions. By this, the MNPs can further be utilized as probes for the physical properties of their molecular environment. In this review, we first investigate the impact of nanoparticle-matrix interactions on magnetic measurements in selected experiments. With these results, we then advanced the imaging modalities magnetorelaxometry imaging and magnetic microsphere tracking to spatially resolve particle-matrix interactions.
\end{abstract}

Keywords: AC susceptibility; magnetic nanoparticles; magnetic particle spectroscopy; magnetorelaxometry; multicolor imaging; particle-matrix interactions.

*Corresponding author: Maik Liebl, Physikalisch-Technische Bundesanstalt, Abbestraße 2-12, 10587 Berlin, Germany, E-mail: maik.liebl@ptb.de

Dietmar Eberbeck and Frank Wiekhorst, Physikalisch-Technische Bundesanstalt, Abbestraße 2-12, 10587 Berlin, Germany

Annelies Coene, Department of Electromechanical, Systems and Metal Engineering, Ghent University, 9052 Ghent, Belgium; and Cancer Research Institute Ghent (CRIG), 9000 Ghent, Belgium

Jonathan Leliaert, Department of Solid State Sciences, Ghent University, 9000 Ghent, Belgium Philine Jauch and Stefan G. Mayr, Leibniz Institute of Surface Engineering (IOM), Permoserstrasse 15, 04318 Leipzig, Germany; and Division of Surface Physics, Department of Physics and Earth Sciences, University of Leipzig, Linnéstrasse 5, 04103 Leipzig, Germany

Margarita Kruteva, Jülich Centre for Neutron Science (JCNS-1) and Institute for Complex Systems (ICS-1), Forschungszentrum Jülich GmbH, Leo-Brandt-Straße, 52425 Jülich, Germany

Lisa Fruhner, Jülich Centre for Neutron Science (JCNS-1) and Institute for Complex Systems (ICS-1), Forschungszentrum Jülich GmbH, Leo-Brandt-Straße, 52425 Jülich, Germany; and Institute of Physical Chemistry, RWTH Aachen University, Landoltweg 2, 52056 Aachen, Germany

Lester Barnsley, Forschungszentrum Jülich GmbH, Jülich Centre for Neutron Science at MLZ, Lichtenbergstr. 1, 85748 Garching, Germany

Ә Open Access. ( 2021 Maik Liebl et al., published by De Gruyter. (c) BY-NC-ND This work is licensed under the Creative Commons Attribution-NonCommercial-NoDerivatives 4.0 International License.

This article has previously been published in the journal Physical Sciences Reviews. Please cite as: M. Liebl, D. Eberbeck, A. Coene, J. Leliaert, P. Jauch, M. Kruteva, L. Fruhner, L. Barnsley,S. G. Mayr and F. Wiekhorst "Magnetic measurement methods to probe nanoparticle-matrix interactions" Physical Sciences Reviews [Online] 2021, 6. DOI: 10.1515/psr-2019-0112 | https:// doi.org/10.1515/9783110569636-009 


\subsection{Magnetic measurement methods to support biomedical applications of magnetic nanoparticles}

Magnetic nanoparticles (MNPs) used in biomedical applications typically consist of a magnetic iron oxide (e.g., magnetite, maghemite) core of 4-30 nm in diameter surrounded by an organic shell. Their unique magnetic properties and the small size of the MNPs allow them to function at a cellular level, making them attractive candidates for cell labeling, imaging, tracking and as carriers. MNPs serve as actuators and local probes in novel therapeutic and diagnostic applications (for review see e.g., [1-4]). For instance, in magnetic hyperthermia an alternating magnetic field is applied toward the MNPs for local heating of tumor tissue. In magnetic drug targeting, the MNPs serve as drug carriers that are remotely guided toward a tumor by magnetic field gradients to accumulate the drug on the tumor side. Other examples are targeted accumulation of genetic material (magnetofection) and contrast agents for magnetic resonance imaging and magnetic particle imaging (MPI).

In all these applications, the MNPs get into contact and thus interact with a biological environment or the surrounding physiological matrix such as tissue, cells, or the bloodstream. The safety and success of these applications vitally depends on a quantitative and spatially resolved knowledge of the interactions attributed to the biological environment. This can be accomplished by probing the surrounding matrix via magnetic measurement techniques capable of tracking changes in the magnetic behavior of MNPs. Examples are the investigation of cellular MNP uptake in target cells [5-7], MNP triggered cell growth [8, 9], the long-term fate and metabolism of MNPs [10], MNP-matrix interactions affecting hyperthermia application $[11,12]$, the interaction of MNPs with blood molecules [13, 14], biological matrices [15] and the passage of MNPs through cell barriers [16-19].

One possibility to analyze particle-matrix interactions (PMIs) is by probing the dynamics of the MNP magnetic moments. The ensemble magnetization responds with a time delay to a change in an external magnetic field. For immobilized MNPs, this delay is determined by the Néel relaxation time [20] necessary for the magnetic moments to overcome the energy barriers resulting from magneto-crystalline structure and/or shape anisotropy. If the MNPs are embedded in a matrix but are still free to mechanically rotate as a whole, Brownian relaxation [21] is possible. The Brownian relaxation time depends on the viscosity of the matrix and the hydrodynamic MNP size distribution. If both relaxation mechanisms are present, the faster mechanism dominates the resulting effective MNP relaxation.

In the following, we describe some magnetic measurements probing the rotational MNP motion to investigate PMIs for biomedical applications. In Section 9.2, we introduce the magnetic measurement techniques used to characterize MNP-matrix systems. 
These are applied in Section 9.3 on well-defined MNP-matrix systems to investigate the effect of PMIs in the respective MNP response. In Sections 9.4 and 9.5, we incorporate these results into the development of novel imaging modalities for a quantitative and spatially resolved description of PMIs.

\subsection{Magnetic measurement and imaging techniques}

\subsubsection{Magnetorelaxometry}

In magnetorelaxometry (MRX) [22], the MNP response to a fast change in an applied magnetic field is detected by a sensitive magnetic field sensor, e.g., a Superconducting Quantum Interference Device (SQUID) [22] or a fluxgate [23]. MRX measurements consist of two consecutive phases. In a first magnetizing phase, a static magnetic field $\mathbf{H}_{v}$ with a typical amplitude of $1 \mathrm{mT} / \mu_{0}$ is applied toward the MNP sample at position $\mathbf{r}_{v}$ in order to partially align the individual particle moments along the field direction resulting in a net magnetic moment $\mathbf{m}_{0}=\chi_{m} \mathbf{H}_{v} X_{\mathrm{MNP}}$. This moment depends on the mass susceptibility $\chi_{m}\left(\mathrm{~m}^{3} / \mathrm{kg}\right)$ of the MNPs, the strength of the applied magnetic field $\mathbf{H}_{v}$ and the MNP mass $X_{\mathrm{MNP}}$ within the sample. In the subsequent measurement phase initiated by switching off the applied magnetic field, the decay of this net magnetic moment over time is detected. This decay is determined by Néel [20] and Brownian relaxation [21] processes and can be described as $\mathbf{m}(t)=\mathbf{m}_{0} \cdot \kappa(t)$ where the monotonically decreasing relaxation function $\kappa(t)$ displays values between one and zero. Both relaxation mechanisms are characterized by a specific time constant:

$$
\tau_{B}=\frac{3 \eta V_{\mathrm{h}}}{k_{\mathrm{B}} T}, \tau_{N}=\tau_{0} \exp \left(\frac{K V_{\mathrm{c}}}{k_{B} T}\right)
$$

The Brownian relaxation time $\tau_{B}$ is determined by the hydrodynamic volume $V_{\mathrm{h}}$ of the nanoparticle, the local viscosity $\eta$ of the particle surroundings, and its thermal agitation given by temperature $T$ and Boltzmann constant $k_{\mathrm{B}}$. The Néel relaxation time is defined by a time constant $\tau_{0}$ with values in literature between $10^{-8}$ and $10^{-12} \mathrm{~s}$ [24], the anisotropy constant $K$ and the volume of its magnetic core $V_{\mathrm{c}}$. If both relaxation mechanisms occur, the particle relaxation is determined by an effective relaxation time $\tau_{\text {eff }}$

$$
\tau_{\text {eff }}\left(V_{c}, V_{h}\right)=\frac{\tau_{N}\left(V_{\mathrm{c}}\right) \tau_{B}\left(V_{\mathrm{h}}\right)}{\tau_{N}\left(V_{\mathrm{c}}\right)+\tau_{B}\left(V_{\mathrm{h}}\right)}
$$

in which the fastest relaxation mechanism dominates the effective relaxation time. In practice, MNP systems exhibit a broad size distribution $P\left(V_{\mathrm{c}}, V_{\mathrm{h}}\right)$. Hence, the relaxation function $\kappa(t)$ detected in MRX experiments is composed of effective relaxation times given by both the core and hydrodynamic MNP size distribution. 
In the MRX measurement phase, the decaying net magnetic moment $\mathbf{m}(t)$ of a MNP source at position $\mathbf{r}_{v}$ gives rise to the MRX signal $B_{s}(t)$ in sensor $s$. The measured flux density of a point-like MNP source, $B_{s}(t)$ detected by sensor $s$ at position $\mathbf{r}_{s}$ with a sensitive axis $\mathbf{n}_{s}=\left[n_{x}, n_{y}, n_{z}\right]$ (normal vector of the sensor) is given by [25]:

$$
B_{S}(t)=\frac{\mu_{0}}{4 \pi}\left(\frac{\left.3\left(\mathbf{n}_{s}^{\mathrm{T}}\left(\mathbf{r}_{s}-\mathbf{r}_{v}\right)\right)\left(\mathbf{r}_{s}-\mathbf{r}_{v}\right)^{\mathrm{T}}\right)}{\left\|\left(\mathbf{r}_{s}-\mathbf{r}_{v}\right)\right\|^{5}}-\frac{\mathbf{n}_{s}^{\mathrm{T}}}{\left\|\left(\mathbf{r}_{s}-\mathbf{r}_{v}\right)\right\|^{3}}\right) \mathbf{H}_{v} \chi_{m} \kappa(t) X_{M N P}
$$

where $\mu_{0}$ is the vacuum permeability and $\mathbf{H}_{v}$ is the magnetic field on position $\mathbf{r}_{v}$. The obtained MRX curve can be parametrized by the relaxation amplitude $\Delta B\left(=B\left(t_{1}\right)-B\left(t_{2}\right)\right.$ with $t_{1}<t_{2}$ ) and the relaxation time $t_{1 / e}$, i.e. the period after that $\kappa(t)$ has dropped by $36.7 \%$ of its value at $B\left(t_{1}\right)$. The relaxation amplitude is directly proportional to the MNP amount of the sample allowing for MNP quantification. The relaxation time parameter $t_{1 / e}$ depends on the Brownian and Néel relaxation processes which will be employed to probe interactions between the nanoparticles and the physiological samples environment.

\subsubsection{Magnetorelaxometry imaging}

Magnetorelaxometry imaging (MRXI) is a sensitive and specific imaging modality for 3D quantification of MNP distributions. In MRXI, the MRX response of a MNP sample is measured on different locations by a sensor array [26, 27], where the spatial encoding is improved by applying a series of spatially constrained magnetic fields using a number of $K$ excitation coils surrounding the measurement volume [25, 28, 29]. By combining all individual MRX measurements, the quantitative 3D reconstruction of the MNP distribution can be obtained as a solution of an inverse problem.

In classical MRX imaging, the presence of only one type of MNPs in the measurement volume is assumed, corresponding to one relaxation function $\kappa(t)$. Note that the only time dependency in the model of Equation (9.3) relies in $\kappa(t)$. It becomes static when only the difference in amplitude $\kappa\left(t_{1}, t_{2}\right)=\kappa\left(t_{1}\right)-\kappa\left(t_{2}\right)$ with $t_{1}<t_{2}$ is of interest. Thus, the classical MRXI forward model calculates only the difference in amplitude between two time points, $t_{1}$ and $t_{2}$, of the MRX signal $B s\left(t_{1}, t_{2}\right)$. In practice, the relaxation product $\chi_{m} \kappa\left(t_{1}, t_{2}\right)$ is obtained from an MRX measurement of a reference sample containing a known MNP amount.

Equation (9.3) can be simplified by including previous MNP material and geometry parameters of the MRXI setup in the sensitivity coefficient $L$ sv linking the particle mass in voxel $v$ to the measurement in sensor $s$

$$
B_{s}\left(t_{1}, t_{2}\right)=L_{s v} X_{\mathrm{MNP}, v}
$$


Equation (9.4) can be extended from just one sensor and one voxel to $S$ sensors and $V$ voxels:

$$
\mathbf{B}=\left[B_{1}, \ldots, B_{S}\right]^{\mathrm{T}}=\sum_{v=1}^{V} L_{s, v} X_{M N P, v}=\mathbf{L X}_{\mathrm{MNP}}
$$

with the sensitivity matrix $\mathbf{L}$ having dimensions $(S \times V)$ and $\mathbf{X}_{\mathrm{MNP}}$ being a vector containing the MNP masses for each voxel $v\left[X_{\mathrm{MNP}, 1}, \ldots, X_{\mathrm{MNP}, V}\right]^{\mathrm{T}}$. In practice, the MRX measurements (Equation 9.5) are performed for $K$ spatially varying magnetic fields generated by the excitation coils. This results in the following forward model

$$
\mathbf{B}_{\text {sim }}=\left[\mathbf{B}_{1}, \ldots, \mathbf{B}_{K}\right]^{\mathrm{T}}=\left[\mathbf{L}_{1}, \ldots, \mathbf{L}_{K}\right]^{\mathrm{T}} \mathbf{X}_{\mathrm{MNP}}
$$

with $\mathbf{B}_{k}$ containing the $S$ relaxation measurements for the $k$-th magnetic field pattern and $\mathbf{L}_{k}$ the corresponding sensitivity matrix. In our MRXI setup 30 excitation coils are applied sequentially to magnetize the MNP distribution with 30 spatially distinct magnetic fields. Thus, we obtain $K=30 \times S$ single MRX measurements and a sensitivity matrix $\mathbf{L}$ of dimension $(K S \times V)$. The MNP distribution consisting of $V$ MNP masses $\mathbf{X}_{\mathrm{MNP}}$ is then recovered by solving

$$
\boldsymbol{X}_{\mathrm{MNP}}^{*}=\underset{\mathbf{X}_{\mathrm{MNP}}}{\operatorname{argmin}}\left\|\mathbf{B}_{\mathrm{sim}}-\mathbf{B}_{\text {meas }}\right\|
$$

where the difference between the modeled measurements $\mathbf{B}_{\text {sim }}$, and the actual MRX measurements $\mathbf{B}_{\text {meas }}$, are minimized by searching for the most probable MNP distribution $\mathbf{X}_{\mathrm{MNP}}$ to cause $\mathbf{B}_{\text {meas }}$. Equation (9.7) has been successfully solved in previous work using a non-negative least squares (NNLS) [30] and by truncated singular value decomposition (tSVD) [26, 29]. In NNLS, Equation (9.7) is iteratively solved with the constraints that all elements of the solution (i.e. the MNP amounts) are positive [31]

$$
\mathbf{w}=\mathbf{L}^{\mathrm{T}}\left(\mathbf{B}_{\text {sim }}-\mathbf{B}_{\text {meas }}\right)
$$

In this equation, $\mathbf{w}$ is the dual vector and should finally only contain elements smaller than or equal to zero, so that the solution $\mathbf{X}_{\mathrm{MNP}}^{*}$ only has positive elements.

\subsubsection{MNP response to alternating magnetic fields}

In contrast to the static magnetic field applied in MRX magnetization, PMIs can also be characterized by the MNP response to alternating magnetic fields. For moderate field amplitudes $\left(0.5-1 \mathrm{mT} / \mu_{0}\right)$ this is referred to as AC susceptibility (ACS). ACS uses an induction coil system to measure the linear dynamic magnetic response of a sample to an alternating magnetic field within the frequency range $10 \mathrm{~Hz}-0.5 \mathrm{MHz}$. At these moderate magnetic field amplitudes, ACS data is well described by the Debye model [32], where 


$$
\chi^{\prime}(\omega)=\frac{\chi_{0}}{1+\left(\omega \tau_{\text {eff }}\right)^{2}}
$$

and

$$
\chi^{\prime \prime}(\omega)=\chi_{0} \frac{\omega \tau_{\text {eff }}}{1+\left(\omega \tau_{\text {eff }}\right)^{2}}
$$

are the real $\chi^{\prime}(\omega)$ and imaginary parts $\chi^{\prime \prime}(\omega)$ of the complex susceptibility $\chi(\omega)$. The equilibrium susceptibility $\chi_{0}$ is given by

$$
\chi_{0}=\frac{\mu_{0} n_{p} \mu_{p}^{2}}{3 k_{\mathrm{B}} T}
$$

with $\mu_{p}$ the moment of a single particle and $n_{p}$ the number of particles. The phase $\varphi$ of the complex susceptibility is defined by $\varphi=\arctan \left(\chi^{\prime \prime}(\omega) / \chi^{\prime}(\omega)\right)$. Note that the effective relaxation time $\tau_{\text {eff }}$ is determined as the maximum in the imaginary part $\omega \tau_{\text {eff }}=1$. Similar to MRXI, additional spatial encoding in ACS by multiple sensors or excitation coils allows for the development of MNP imaging approaches [33-36].

Increasing the amplitude of the AC magnetic field allows to obtain additional signal contributions of the MNPs by their nonlinear magnetic susceptibility. This is employed in Magnetic Particle Spectroscopy (MPS). Here, the MNP response to AC magnetic fields of some tens of $\mathrm{mT} / \mu_{0}$ (here $B=25 \mathrm{mT}$ ) at fixed frequency in the $\mathrm{kHz}$ range (here $f_{0}=25 \mathrm{kHz}$ ) is detected by an induction coil system. The MPS signal does not only contain signal components at the excitation frequency $f_{0}$, but also MNP-specific higher harmonics (i.e. odd multiples of $f_{0}$ ). After obtaining the MPS spectrum from a Fourier transformation, two characteristic parameters can be extracted. These are the amplitude of the third harmonic $M_{3}$ and the harmonic ratio $M_{5} / M_{3}$, i.e. amplitude ratio between fifth and third harmonic. $M_{3}$ is directly proportional to the MNP amount of the sample. $M_{5} / M_{3}$ depends on the dynamic magnetic behavior of the MNPs and is often used as indicator for PMIs [12]. In magnetic particle imaging (MPI) [37], this concept is combined with a spatial encoding by magnetic gradient fields for the 3D quantitative imaging of MNP distributions.

\subsection{Interaction of MNPs with matrices}

Nanoparticle matrix systems generally consist of MNPs embedded in a nonmagnetic often soft material environment. Prominent technical applications are MNP-polymer systems where mechanical properties of the matrix like the elastic moduli shall be controlled by external magnetic fields (e.g., [38-40]). In a biological MNP-matrix system the MNP can be located in the cellular system (inside the cell, at the cell surface or in the intercellular space) and may be targeted to transport a drug by external magnetic fields. Since the magnetic response of the MNPs is sensitive to the particle surrounding, this offers a way to analyze PMIs. To reduce the complexity of biological 
systems, one often resorts to model systems like MNPs embedded in hydrogel (also called ferrogel). Here, the viscous hydrogel emulates the biological matrix with welldefined properties. Microscopically, the hydrogel is not a homogeneous matrix, but a polymer scaffold filled with an aqueous, solvating fluid.

The PMIs in hydrogels are mainly caused by (i) the MNP binding with the polymer scaffold and (ii) the drag of MNP movements determined by the (local) viscosity of the solvating fluid and the inner structure of the hydrogel, e.g., the pore size and its connectivity. On the other hand, the coating and the hydrodynamic size of the MNPs are important factors to study PMIs. For instance, the presence of functional groups like carboxylate groups $\left(\mathrm{COO}^{-}\right)$in the coating may lead to a preferential binding of MNPs to positively charged domains of the hydrogel scaffold. The drag of the MNP motion is caused by the fluid resistance, which is mainly determined by the hydrodynamic size of the MNPs but also by reversible (weak) bonding caused by macromolecular entanglement between coating molecules and matrix molecules. The binding functionality can be measured using a magnetic assay [41], but in the following we focus on the physical structure of the coating, i.e. its thickness and density.

As magnetic measurement techniques we apply MRX, ACS and MPS (Section 9.2) and analyze PMIs by detecting changes in the Brownian and Néel relaxation of the MNPs. We used MNP systems with different core material and coating. For convenience they are labeled $\mathrm{X}_{i}^{\star} \mathrm{Y}$, where the first letter $\mathrm{X}$ indicates the core material, with $\mathrm{X}=\mathrm{M}$ for magnetite and $\mathrm{X}=\mathrm{C}$ for cobalt ferrite, followed by a consecutive sample number $i$. The term $\mathrm{Y}$ after the delimiting * denotes the key molecular part of the coating. Here, $\mathrm{Y}=\mathrm{COO}^{-}$indicates carboxylate groups, $\mathrm{Y}=\mathrm{CONH}-\mathrm{PEG}$ denotes amino-PEG (polyethylene-glycol) coupled to a carboxylate group and, $\mathrm{Y}=\mathrm{S}$ stands for silica $\left(\mathrm{SiO}_{2}\right)$.

\subsubsection{Probing the melting of a matrix by Brownian MNP relaxation}

First, we investigated the melting behavior of ferrogels in gelatin. Here, we used the Brownian relaxation of the MNPs to probe the local viscosity of the matrix during its melting. To this end we used MPS and MRX as sensitive magnetic measurement techniques to detect variations in the MNP relaxation caused by matrix property changes during the melting (e.g., disintegration, reduced viscosity).

Figure 9.1 displays the MRX relaxation time and the MPS amplitude of the third harmonic normalized to iron mass $M_{3}$ during the melting process of gelatin, i.e. as a function of temperature. As expected, significant parameter changes are observed for temperature close to the gelatin melting point of about $308 \mathrm{~K}$. This is attributed to the onset of Brownian rotation of the MNPs becoming possible above the melting point. Interestingly, there are small parameter changes already before the macroscopic melting point (Figure 9.1). These are interpreted as local melting [42], where the MNPs sense a maceration of the matrix without being disintegrated. This is further confirmed by a drop in the MRX relaxation amplitude (not shown in the graph). 


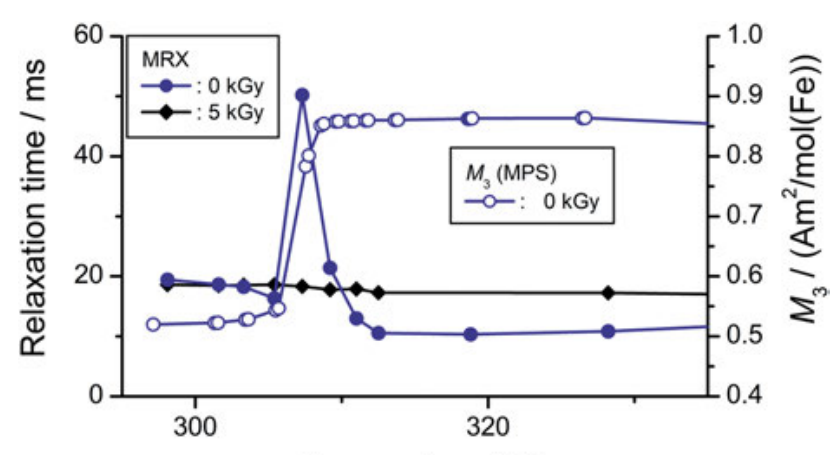

Temperature / K

Figure 9.1: MPS third harmonic $M_{3}$ (open blue circles) and MRX relaxation time (closed blue symbols) during melting of gelatin with embedded MNPs. Black curve: Relaxation time after hardening of the ferrogel by electron irradiation with $5 \mathrm{kGy}$ dose protecting the gelatin from melting up to at least $70^{\circ} \mathrm{C}$. Adapted with permission from Wisotzki et al. [42]. Copyright 2017 Royal Society of Chemistry.

While the $M_{3}$ clearly remains increased after crossing the melting point, the MRX relaxation time reaches a maximum at the melting point. This can be explained by the presence of Brownian and Néel relaxation at the same time: The MNP system exhibits a broad distribution of Néel relaxation times that dominate the magnetic behavior up to the melting point. A fraction of MNPs exhibits Néel relaxation times above the upper limit of the MRX measurement time window what explains the small initial value of the effective relaxation time. In the melting process, particles of those fraction now enter the MRX measurement time window by the Brownian relaxation mechanism, which leads to the maximum in the relaxation time. With increasing temperature, the MNPs sense a strong change from high to low viscosity resulting in a decrease of their viscosity-dependent Brownian relaxation times after the maximum. A more detailed and quantitative interpretation can be found in Ref. [42]. The black squares in Figure 9.1 are the relaxation times obtained from a ferrogel that was hardened by electron beam of strength $5 \mathrm{kGy}$. While no macroscopic melting was observed, small variations in the relaxation times during the temperate increase are again attributed to changes in the local viscosity.

Thus, we demonstrated that MRX and MPS are sensitive to both, the local and macroscopic melting behavior of ferrogels.

\subsubsection{Probing the spatial arrangement of MNPs within a matrix by Néel relaxation}

The Néel relaxation is defined as the thermal activated jump of the MNP magnetic moment over the barrier of its anisotropy energy, i.e. the internal flip of the magnetization vector. If MNPs aggregate, then dipole-dipole interactions between MNPs might 


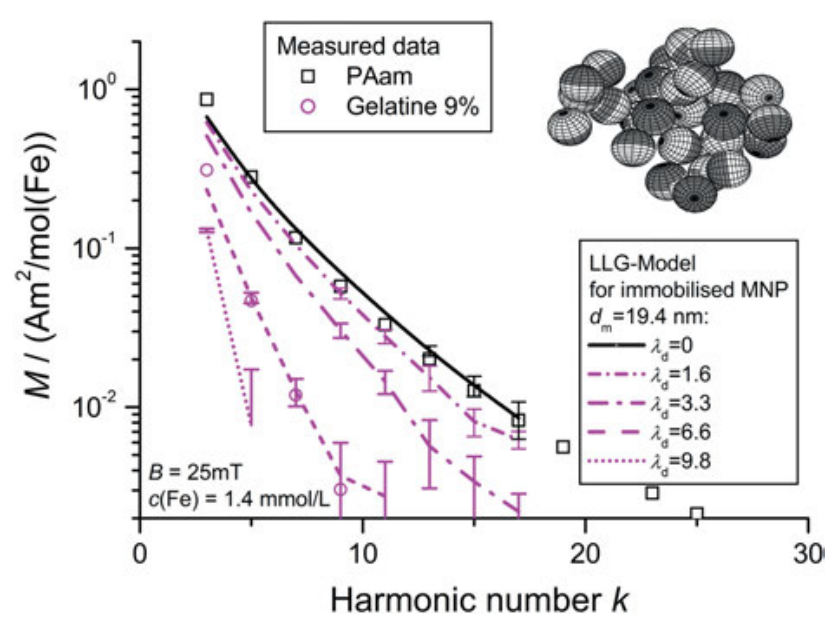

Figure 9.2: MPS-data of ferro-hydrogels on the base of polyacrylamide gel (PAam) and gelatin (symbols) together with the results of the LLG-model for clusters of 30 randomly arranged immobilized MNP of one size $\left(d_{\mathrm{m}}=19.4 \mathrm{~nm}\right)$ taking into account the indicated interaction energies.

alter these internal energy barriers. This affects the magnetic susceptibility detected by ACS and MPS. Hence, changes in ACS or MPS signals provides information about the aggregation state of embedded MNP probes. To investigate these changes, we immobilized magnetite based MNP with a coating containing carboxylate groups on its surface [42] in two different matrices, polyacrylamide (PAam) and gelatin. The MNPs were added prior to the polymerization process to ensure their homogeneous distribution within the sample. After polymerization, the samples were measured by MPS. The observed spectra are depicted in Figure 9.2. The spectrum of the gelatin-based sample decays much faster and the third harmonic $M_{3}$ is only $31 \%$ of that of the PAam based sample.

Using the parameters magnetic moment distribution and minimal possible particle distance, we modeled the MPS spectra of immobile MNPs solving the Landau-LifshitzGilbert (LLG) equation considering the dipole-dipole interactions between the MNPs (in collaboration with P. Ilg, University of Reading). We modeled the magnetization response of close packed clusters comprising 30 randomly arranged MNPs of one size, all having a mean volume diameter of $19.4 \mathrm{~nm}$. A clear dependence of the modeled MPS spectra on the interaction parameter $\lambda_{d}=\mu_{0} \mu^{2} /\left(4 \pi r^{3} k_{\mathrm{B}} T\right)$ is found (Figure 9.2). The interaction parameter $\lambda_{\mathrm{d}}$ describes the dipole-dipole interaction between two magnetic moments $\mu$ at a distance $r$. While the model of noninteracting MNPs $\lambda_{\mathrm{d}}=0$ describes the data of the PAam sample, an interaction parameter $\lambda_{d}=6.6 \mathrm{~nm}$ is necessary for a description of the gelatin-based sample. This interaction parameter corresponds to the interaction energy of closely packed MNPs. Hence, we conclude that the MNPs are aggregated within the gelatin matrix. 
We hypothesize that the observed aggregation is caused by negatively charged carboxylate groups of the MNPs interacting with the charged collagen molecules of the gelatin. While gelatin A is predominantly positively charged $(\mathrm{pI}=8.5-9)$ PAam does not contain charged functional groups. Note, due to hydrolysis there may be a molecular content of carboxylate groups of about $2-6 \%$.

\subsubsection{Requirements on MNPs to probe particle-matrix interactions}

\subsubsection{Suppressing Néel relaxation to sensitively monitor the MNP rotation inside a matrix}

The physical state of the matrix (e.g., soft, hard, charged) can be characterized by measuring the Brownian motion of embedded MNPs. Methods like ACS, MRX, and MPS are very sensitive to detect rotational MNP movement while a translational motion requires magnetic gradient fields to drag the MNPs through the matrix. To accurately measure the rotational motion of the MNP as a whole, the Néel relaxation process (i.e. the rotation of the magnetic moment within the MNP) should be suppressed by a sufficiently high anisotropy energy barrier, i.e. $\tau_{\mathrm{N}} \gg \tau_{\mathrm{B}}$. Cobalt ferrite is an appropriate core material for such MNPs because its anisotropy constant is about 10 times higher than that of magnetite. Furthermore, translational motion requires a high saturation magnetization of the whole core-shell MNP and the overall size of the MNP should match the structure size (pore size) under study. Finally, the analysis of dipolar interactions (Section 9.3.2) requires MNPs with high magnetic moments and a thin coating layer.

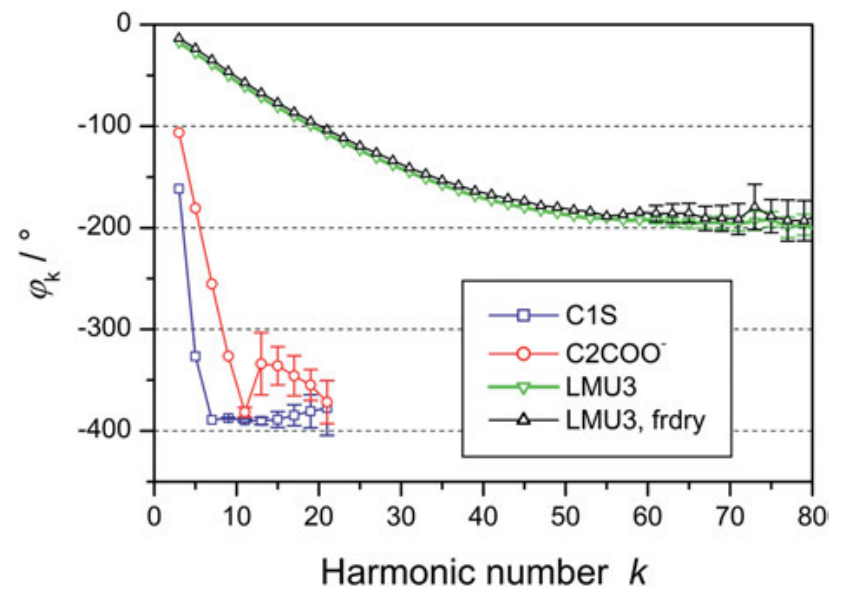

Figure 9.3: MPS phase data of the cobalt ferrite systems $\mathrm{C} 1{ }^{\star} \mathrm{S}$ and $\mathrm{C}^{\star}{ }^{\star} \mathrm{COO}^{-}$in comparison to magnetite based magnetosome particles LMU3 [43] dispersed in water and after freeze drying within a mannitol matrix. 
The prevalence of the dominating relaxation mechanism (Brownian or Néel) can be assessed by the phase $\varphi$ of the MPS data. For MNP systems with dominating Néel relaxation it is often observed that the MPS phase smoothly decays with the number of harmonics $k$ asymptotically approaching to $-180^{\circ}$ (Figure 9.3). Such a characteristic phase curve is shown for mobile and immobilized magnetite based magnetosome particles LMU3 [43] in Figure 9.3. On the other hand, the Néel relaxation of cobalt ferrite MNPs with a core diameter larger than about $12 \mathrm{~nm}$ is strongly suppressed. Therefore, the Brownian relaxation dominates. The MPS phase curve of these MNPs decline nearly linearly and much faster with phases far below $-180^{\circ}$ for higher harmonics. The phase saturates at harmonic numbers, since here the signal of Néel relaxation of small MNPs prevails that of Brownian relaxation.

\subsubsection{Opsonization as consequence of MNP-biomolecule interactions}

The coating of MNPs influences their functionality and clearance in biomedical applications. The properties of the coating (thickness, density) might further be influenced by the medium in which the MNPs are dispersed. A prominent example is the opsonization of MNPs by proteins that may occur in blood and determines the interaction of the MNPs with the organism. Here, we combined the information gained by the magnetic measurement method ACS and Small Angle Neutron Scattering (SANS) to probe the opsonization of MNPs by the thickness and density of their opsonization layer. The thickness of the MNP coating can be estimated from the difference between hydrodynamic diameter $d_{\mathrm{h}}$ and core diameter $d_{\mathrm{c}}$. MRX and ACS are promising integral measurement methods for the estimation of the distribution of $d_{\mathrm{h}}$ [44, 45]. The complex susceptibility $\chi(\omega)$ as measured by ACS is well suited to estimate $d_{\mathrm{h}}$. We modeled $\chi(\omega)$ of noninteracting MNP with a lognormal core diameter distribution, $f\left(d_{\mathrm{c}}\right)$, assuming each MNP surrounded by a shell of thickness $\delta_{\mathrm{s}}$

$$
\chi(\omega)=\frac{1}{\overline{d_{\mathrm{c}}^{3}}} \int \frac{f\left(d_{\mathrm{c}}\right) d_{\mathrm{c}}^{3} L\left(H, M_{\mathrm{s}}, d_{\mathrm{c}}, T\right) \chi_{0}}{1-i \omega \tau_{\mathrm{eff}}} d_{\mathrm{c}}
$$

Here, $M_{\mathrm{s}}$ denotes the saturation magnetization and $L$ the Langevin function. The hydrodynamic volume is given by $V_{\mathrm{h}}=\pi\left(d_{\mathrm{c}}+\delta_{\mathrm{s}}\right)^{3} / 6$. This becomes of particular interest when measuring in a (biological) environment. We estimated the thickness of the MNP's shell, $\delta_{\mathrm{s}}$, fitting the model of Equation (9.12) to $\chi(\omega)$.

Small Angle Neutron Scattering (SANS) with contrast variation provides a very sensitive access to the thickness of the MNP coating [46-48]. The scattering intensity of the sample scales with the square of the difference of scattering length densities of the particle core, $\rho_{\mathrm{p}}$, the particle's shell, $\rho_{\mathrm{s}}$, and that of the whole sample, $\rho_{0}$. Here, we varied $\rho_{0}$ by replacing $\mathrm{H}_{2} \mathrm{O}$ by heavy water $\mathrm{D}_{2} \mathrm{O}$. In $\mathrm{H}_{2} \mathrm{O}$ the contrast is mainly determined by the nanoparticle core scattering and in $\mathrm{D}_{2} \mathrm{O}$ the details of shell structure are seen (see, e.g., [49]). The SANS measurement were performed on the instrument KWS-1 
a

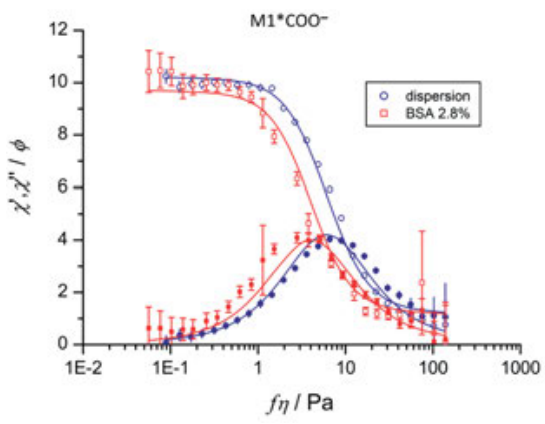

b

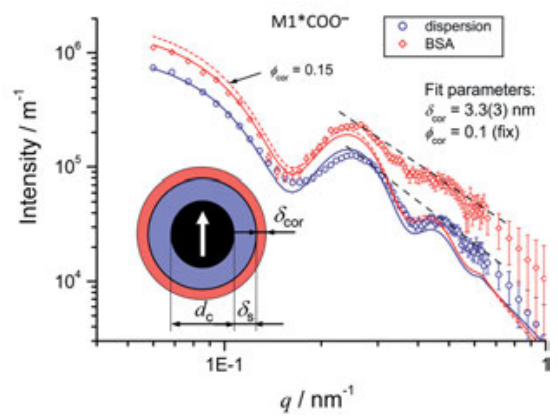

Figure 9.4: ACS-data (left) and SANS-data (right) of the commercially available nearly monodisperse MNP system SHP-30, denoted as $\mathrm{M}^{\star}{ }^{\star} \mathrm{COO}^{-}$, before (blue symbols) and after opsonization by BSA (red symbols). The chosen for ACS delineation already eliminates the effect of the sample viscosity $\eta$ on the data. The lines represent the best fit of a model of lognormally distributed magnetite cores with a coating and a corona layer of one thickness (inset). The short dash line represents the best fit model with corona with an adjusted density volume fraction of the corona, $\phi_{\text {cor }}$, illustrating the sensitivity of SANS with respect to $\phi_{\text {cor }}$. The SANS data of the sample with the opsonized MNP (in BSA) suffers from a quite large systematic uncertainty because of the high intensity of the background scattering of the BSA-solution. A solvation layer thickness of $1.5 \mathrm{~nm}$ was assumed in the fit. $\phi$ and $\eta$ denote the volume fraction of core material, here magnetite, and the viscosity of the sample, respectively.

$[50,51]$ at the MLZ in Garching, Germany. The incident neutron wavelength $\lambda$ was $7 \AA$ $(\Delta \lambda / \lambda=10 \%)$. Data was obtained from two different detector and collimation distances: the detector distances of 1.5 and $8 \mathrm{~m}$ and a collimation distance of $8 \mathrm{~m}$ leading to a total $Q$ range of $0.05-1.5 \mathrm{~nm}^{-1}$. The samples were measured in quartz cells with beam path of $1 \mathrm{~mm}$ for $\mathrm{H}_{2} \mathrm{O}$ and $2 \mathrm{~mm}$ for $\mathrm{D}_{2} \mathrm{O}$. The measurements were done at room temperature. The size of the sample aperture was set as $6 \times 6 \mathrm{~mm}$. The data presented here were converted to an absolute intensity unit of $\mathrm{m}^{-1}$ taking into account the sample thickness, transmission, the scattering from a standard sample and the background from electronic noise, the solvent and the quartz cell. Data reduction has been done using the QtiKWS software [52].

Fitting a model $[53,54]$ describing the SANS data by noncorrelated core-shell particles (Figure 9.4) with the corresponding scattering length densities, we could estimate the thickness $\delta_{\mathrm{s}}$ and density $\phi_{\mathrm{s}}$ of the polymeric shell of the MNP system $\mathrm{M} 1^{\star} \mathrm{COO}^{-}$to $\delta_{\mathrm{s}}=2.9(1) \mathrm{nm}$ and $\phi_{\mathrm{s}}=1.0$ (2). Note, that only particles with a very narrow size distribution make the analysis robust (Figure 9.4b).

The particles $\mathrm{M}^{\star}{ }^{\star} \mathrm{COO}^{-}$were dispersed in a $4.3 \%$ bovine serum albumin (BSA) solution to probe their opsonization, i.e. a corona formation of BSA around the MNPs increasing their hydrodynamic diameter. For the estimation of the thickness of the second layer, i.e. the opsonization layer, we combined SANS and ACS data using a common model where both submodels share the same fit parameters of the particle 
Table 9.1: Mean volume core diameter, $d_{\mathrm{cv}}$, thickness of the coating shell, $\delta_{\mathrm{s}}$, and the thickness of the corona, $\delta_{\text {cor }}$, obtained by fitting analysis of ACS, $\mathrm{M}(\mathrm{H})$, and additionally for $\mathrm{M}^{\star}{ }^{\star} \mathrm{COO}^{-}$, SANS data.

\begin{tabular}{|c|c|c|}
\hline Quantity & $\mathrm{M} 1^{*} \mathrm{COO}^{-}$ & $\mathrm{M} 2^{\star} \mathrm{COO}^{-}$ \\
\hline$d_{\mathrm{cv}} / \mathrm{nm}$ & $30.3 \pm 0.2$ & $30.0 \pm 0.2$ \\
\hline$\delta_{\mathrm{s}} / \mathrm{nm}$ & $2.9 \pm 0.1$ & $2.8 \pm 0.4$ \\
\hline$\delta_{\text {cor }} / \mathrm{nm}$ & $3.3 \pm 0.3$ & $4.2 \pm 0.2$ \\
\hline
\end{tabular}

structure (Figure 9.4). This was reasoned because of the relatively weak signal change in SANS by the opsonization and a relatively large uncertainty in SANS due to the large background signal. The fit yields an opsonization layer thickness of $\delta_{\text {cor }}=3.3(3) \mathrm{nm}$ (Table 9.1). This matches well with the shortest dimension of an BSA molecule the shape of which is assumed similar to an oblate ellipsoidal shape with axes of $(3.4 \times 8.4 \times 8.4) \mathrm{nm}^{3}[54]$.

The relatively small signal increase measured following the opsonization (Figure 9.4b) reflects the low density (or volume fraction) of the corona formed by opsonization of about $\phi_{\text {cor }}=0.1$. Note that $\phi_{\text {cor }}$ was adjusted manually because of fitting instabilities caused by the relatively large systematic uncertainties as mentioned above. Also, the applied core-shell-opsonization layer model (Figure 9.4b inset) might be an oversimplification of the real corona structure. For instance, the corona might have an inhomogeneous structure as suggested in [55]. The resulting lower fractal dimensionality would explain the slightly slower decay of $I(q)$ of opsonized MNPs within the Porod regime, as marked by dashed line. It was shown that ACS reliably allows to estimate the thickness of an opsonization layer while SANS additionally yields the density of this layer. By combined evaluation of ACS and SANS data, we could enhance the confidence of the results. In particular, with given thickness of the solvation layer, ACS sensitively displays the increase of the hydrodynamic diameter of the MNPs by the opsonization process.

\subsubsection{Translational motion of MNPs with different coatings in matrices}

To gain information about the migration of MNPs within a biological matrix, we investigate the translational motion of MNPs driven by an external magnetic field gradient through a collagen matrix. To achieve a large magnetic force, we utilized MNPs with a large magnetic moment of about $4 \mathrm{aAm}^{2}$. The MNPs were similar to those used in Ref. [42]. As mentioned above, the capability of the outer MNP shell to bind to the matrix determines the MNP's functionality. Therefore, we used two MNP systems with identical cores but different coatings. The carboxymethyl dextran shell of the first 
Table 9.2: Mean volume core diameter, $d_{\mathrm{cv}}$, thickness of the coating shell, $\delta_{\mathrm{s}}$, and the thickness of the corona, $\delta_{\text {cor }}$, obtained by fitting analysis of ACS and $M(H)$ data.

\begin{tabular}{lr}
\hline Quantity & M2*CONH-PEG \\
\hline$d_{\mathrm{cv}} / \mathrm{nm}$ & $35.6 \pm 0.2$ \\
$\delta_{\mathrm{s}} / \mathrm{nm}$ & $20.5 \pm 0.7$ \\
$\delta_{\mathrm{cor}} / \mathrm{nm}$ & $0.5 \pm 0.7$ \\
\hline
\end{tabular}

system, $\mathrm{M} 2^{\star} \mathrm{COO}^{-}$, presents carboxylate groups leading to a negative zeta potential at neutral $\mathrm{pH}$. The second system, M2*CONH-PEG (Table 9.2), was prepared by coupling amino-PEG to $\mathrm{M}^{\star} \mathrm{COO}^{-}$. Theoretically, no charged functional groups are present in M2*CONH-PEG. This leads to the hypothesis that M2*CONH-PEG can be moved more effective by a field gradient through a hydrogel than $\mathrm{M}^{\star}{ }^{\circ} \mathrm{COO}^{-}$. To verify this, we filled a tubular sample holder with collagen gel and loaded it with MNP dispersions. A homogeneous magnetic field gradient of $8 \mathrm{~T} / \mathrm{m}$ was applied for $48 \mathrm{~h}$. Afterward, the sample holder with the collagen was dissected into nine segments and the MNP content of each collagen segments was quantified by MPS. The results show that the concentration of $\mathrm{M} 2{ }^{\star} \mathrm{CONH}-\mathrm{PEG}$ within the second segment is 2.4 times higher than that of the

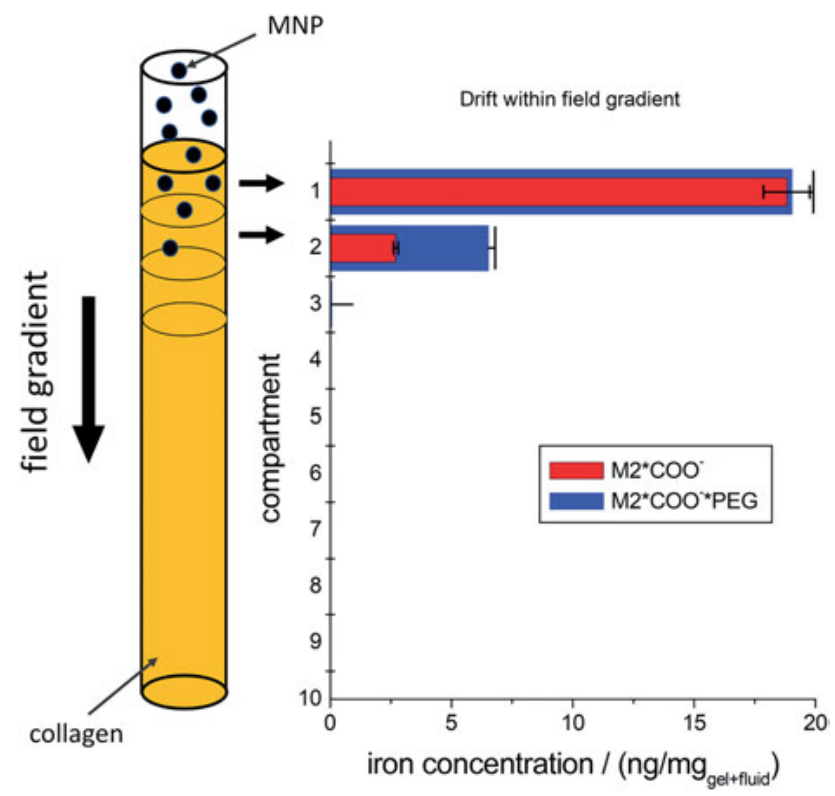

Figure 9.5: Left: Experimental set-up for the drag of MNP through a collagen gel by a magnet field gradient. Right: Concentration of MNP (iron) within each collagen segment (of nine) quantified by MPS. 
corresponding $\mathrm{M} 2^{\star} \mathrm{COO}^{-}$sample (Figure 9.5). Hence, $\mathrm{M}{ }^{\star}{ }^{\star} \mathrm{CONH}-\mathrm{PEG}$ has a substantial higher mobility in collagen than $\mathrm{M} 2^{\star} \mathrm{COO}^{-}$.

Then, we related the observed different mobility in a collagen gel to the opsonization by BSA, justified by the molecular similarity of collagen and BSA. We checked the ability of the MNP to bind the proteins BSA by opsonization measurements using ACS as it was done in Section 9.3.3.2, for $\mathrm{M}^{\star} \mathrm{COO}^{-}$. The fit of Equation (9.12) to the ACS data of samples prior to BSA incubation (Figure 9.6) reveals that the coating of $\mathrm{M} 2{ }^{\star} \mathrm{CONH}-\mathrm{PEG}$ is about $18 \mathrm{~nm}$ thicker than in $\mathrm{M}^{\star} \mathrm{COO}^{-}$ (Table 9.1). Obviously, this is the PEG-layer thickness. After BSA incubation the hydrodynamic size of $\mathrm{M} 2^{\star} \mathrm{COO}^{-}$increase by $4.2 \mathrm{~nm}$ while it does not change for $\mathrm{M} 2^{\star} \mathrm{CONH}-\mathrm{PEG}$ (Tables 9.1 and 9.2). M2*CONH-PEG was also not opsonized, even by fetal calf serum (FCS) albumin which contains, in contrast to BSA, a rich variety of proteins and other molecules. This observation supports the commonly accepted thesis of stealth properties of PEG decorated particles [56].

The apparent absence of any opsonization of $\mathrm{M} 2{ }^{\star} \mathrm{CONH}-\mathrm{PEG}$ correlates well with the observed higher mobility through the collagen gel as shown above. Furthermore, we expected that $\mathrm{M} 2{ }^{\star} \mathrm{CONH}-\mathrm{PEG}$ would have passed through the all gel segments of the tubular sample holder after $48 \mathrm{~h}$ since the large mean pore size of about 100-200 nm of the collagen gel, inferred from reference [57] where a PAam gel of nearly the same composition was investigated by TEM. Hence, it seems to be likely that the $\mathrm{M} 2{ }^{\star} \mathrm{CONH}-\mathrm{PEG}$ mobility is reduced by binding to the collagen matrix.

a

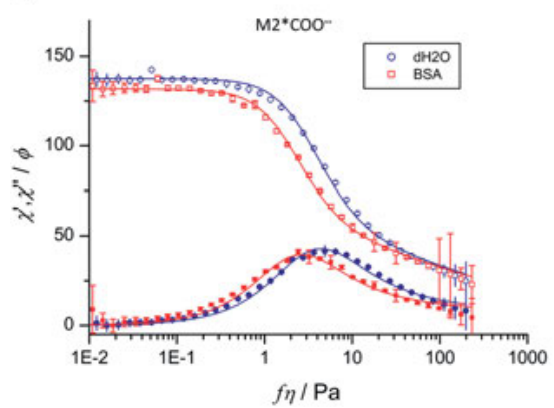

b

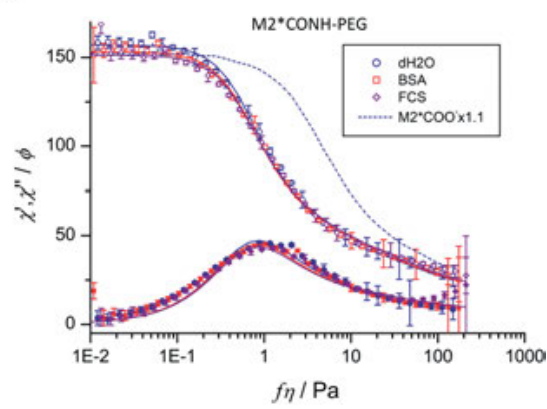

Figure 9.6: ACS-data of the magnetite MNP systems $\mathrm{M}^{*}{ }^{*} \mathrm{COO}^{-}$(a) and $\mathrm{M} 2{ }^{\star} \mathrm{CONH}{ }^{\star} \mathrm{PEG}$ (b) before and after opsonization with BSA and FCS. The data were normalized to the volume fraction of magnetite, $\phi$. A model of noninteracting core-shell MNP was fitted simultaneously to $M(H)$ and ACS-data (lines). The dashed line on the right axes in (b) represents the curve of $\mathrm{M2}^{*} \mathrm{COO}^{-}$before opsonization, with adapted amplitude for better comparison. 


\subsection{Multicolor magnetorelaxometry imaging for quantitative visualization of particle-matrix interactions}

\subsubsection{Classical MRXI and multicolor magnetorelaxometry imaging}

Classical MRXI focuses on the quantitative reconstruction of a spatial MNP distribution. Because the relaxation curve is very sensitive to the direct environment of MNPs, MRXI has been extended to also gain information about interactions between MNPs and their surrounding matrix $[58,59]$. By this approach, called multicolor MRXI, the spatial distribution of MNPs interacting with different molecular environments (e.g., liquid, blood, cells, tissue matrix) or MNPs with distinct properties (e.g., size distributions) can be reconstructed and separated. For convenience, we further summarize such differences as phases. As an example, Figure 9.7 shows classical MRX imaging and the multicolor MRXI approach on two distinct MNP distributions, with the respective MRXI reconstructions in the insets.

Our MRXI setup consists of an MNP distribution phantom, a set of excitation coils on printed circuit boards mounted on top (not shown in Figure 9.7 for clarity) and bottom of the phantom and a 304 SQUID sensor system [60] for MNP relaxation detection. The

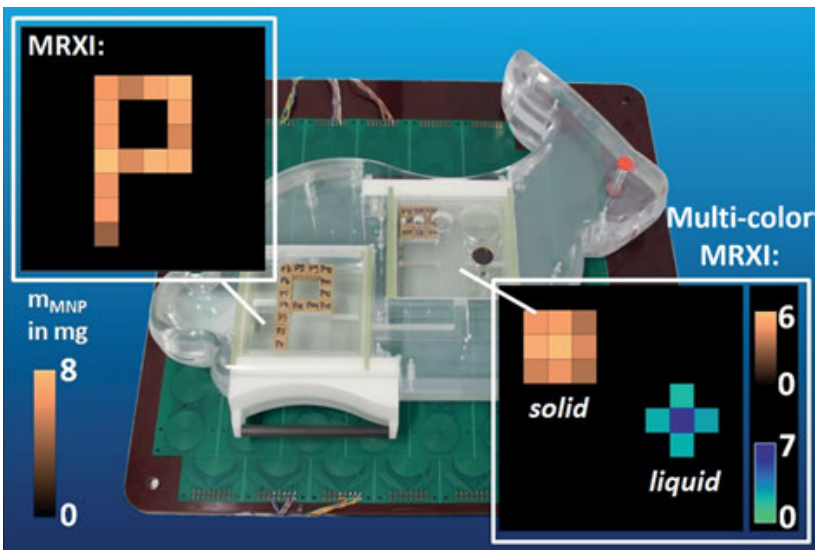

Figure 9.7: MRX imaging using multiple measurements on a rabbit phantom. The MNP phantom models defined MNP distributions with physical and physiological parameters of magnetic drug targeting application in rabbits. Left support: MNP test distribution in the tumor region of the rabbit phantom (brown cubes containing $6 \mathrm{mg}$ of MNP each) and reconstructed MNP distribution after MRX imaging. Right support: multicolor MRXI with MNP support containing $1 \mathrm{~mL}$ MNP suspension (12 mg MNP) and 9 MNP loaded gypsum cubes (brown cubes containing $6 \mathrm{mg}$ of MNP each) and MRXI reconstructions achieved by multicolor MRXI for MNPs in liquid phase (blue-green voxels) and MNPs solid phase (brown voxels). 
phantom has a shape and size comparable to a rabbit and was developed to analyze the performance of MRXI in a magnetic drug targeting study done by Alexiou et al. [61, 62]. The phantom contains two supports, to model the regions-of-interest (ROIs) in the tumor region (left) and the liver region (right) of the rabbit. Each support allows the flexible arrangement of MNP distributions within a total volume of $9.6 \mathrm{~cm} \times 9.6 \mathrm{~cm} \times 6 \mathrm{~cm}$. The circuit boards provide 15 excitation coils above and below each support. The coils are applied sequentially to generate the spatially constrained magnetic fields of the MRXI sequence.

For classical MRXI (which assumes all MNPs in the same phase), we formed in the ROI tumor an MNP distribution resembling the letter "P" out of 15 gypsum cubes (each loaded with $3.7 \mathrm{mg} / \mathrm{cm}^{3}$ MNPs). Here, all MNPs are in a solid phase, i.e. fixed in a gypsum matrix, so that only Néel relaxation contributes. The MRXI reconstruction reveals a high correlation to the nominal MNP distribution (Pearson's correlation coefficient $>95 \%$ ) and a mass deviation of the phantom's total MNP amount below $10 \%$ [58].

In the multicolor MRXI experiment, we used an MNP distribution containing two phases (MNPs in a solid matrix and MNPs suspended in a liquid) arranged in the ROI liver (right support and inset of Figure 9.7). The MNP distribution in the solid phase is formed of nine gypsum cubes (each loaded with $3.7 \mathrm{mg} / \mathrm{cm}^{3} \mathrm{MNPs}$ ) resembling a square and placed beside a $1 \mathrm{~mL}$ MNP suspension in a liquid phase $\left(12 \mathrm{mg} / \mathrm{cm}^{3}\right.$ of MNPs) in a circular container of $2 \mathrm{~cm}$ diameter. For the chosen MNP relaxation type we mainly have Néel relaxation in the region of MNPs in the solid phase and Brownian relaxation in the region of MNPs in the liquid phase. This allows for a quantitative separation of MNPs in both phases in one MRXI measurement as visualized by different colormaps in Figure 9.7 (the brown color corresponds to the MNPs in solid phase and the blue color to the MNPs in the liquid phase). Applying multicolor MRXI, it was possible to simultaneously and quantitatively reconstruct and separate MNPs in both phases forming our MNP distribution with a total mass deviation below 10\% [58]. In the following, we detail our multicolor MRXI approach and extend the phantoms from this two-phase system to multiphase systems [59].

\subsubsection{Extending MRXI to quantitatively imaging particle-matrix interactions}

While classical MRXI employs only the amplitude $\kappa\left(t_{1}, t_{2}\right)$ for image reconstruction, we make use of the complete relaxation curve shape $\kappa(t)$ in our multicolor MRXI approach [59]. This additional temporal information allows to extract portions of distinct MNP relaxation curves due to different phases out of a single MRX measurement. In our approach, the MRXI reconstruction is split up into two subproblems as schematically depicted in Figure 9.8. The first subproblem finds the relative contributions of $N$ distinct MNP relaxation curves $\chi_{n} \kappa_{n}(t)$ ( $N$ distinct phases) to the measured signal in the sensors. 
These are obtained from reference measurements of samples in a specific phase under well-known and controlled conditions. It is assumed that similar phases occur simultaneously in the sample under investigation. The second subproblem finds the associated MNP distribution to the previously determined relative contribution in the sensors. These $N$ distributions are finally merged in a color-coded image in which the color represents the specific phase and the intensity reflects the amount of MNPs. In the reference measurement well-known MNP samples with controlled phase are positioned at location $\mathbf{r}_{v}$ and the MRX signal is normalized to applied magnetic field $\mathbf{H}_{v}$ and MNP mass $X_{\text {ref,n }}$ to obtain $\chi_{n} \kappa_{n}(t)$ for use in the first subproblem:

$$
\left.\chi_{n} \kappa_{n}(t)=\frac{B_{\text {ref }}(t)}{\frac{\mu_{0}}{4 \pi}\left(\frac{\left.\left.3\left(\mathbf{n}_{s}^{\mathrm{T}}\left(\mathbf{r}_{s}-\mathbf{r}_{v}\right)\right)\left(\mathbf{r}_{s}-\mathbf{r}_{v}\right)^{\mathrm{T}}\right)\right)}{\left\|\left(\mathbf{r}_{s}-\mathbf{r}_{v}\right)\right\|^{5}}-\frac{\mathbf{n}_{s}^{\mathrm{T}}}{\left\|\left(\mathbf{r}_{s}-\mathbf{r}_{v}\right)\right\|^{3}}\right.}\right) \mathbf{H}_{v} X_{\text {ref }, n} .
$$

\section{Actual MNP distribution:}
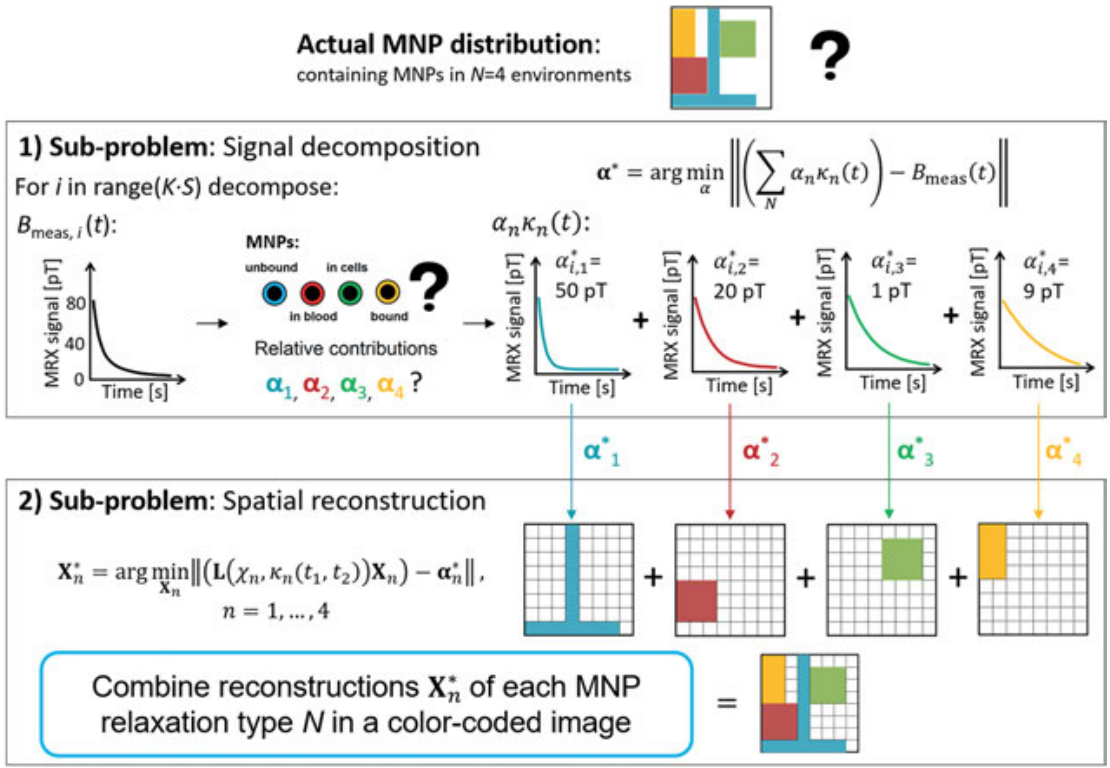

Figure 9.8: Principle of multicolor MRXI. An actual MNP distribution (top) contains $N=4$ compartments where the MNPs are either suspended (blue), dispersed in blood (red), in cells (green) or bound to a fixed matrix (yellow). In a first subproblem, each measured relaxation signal $B_{\text {meas }}, i(t)$ of the MRXI sequence is decomposed into portions of four reference MNP relaxations $\alpha_{n} K_{n}(t)$, recorded on MNP samples of known MNP mass and in the respective environment. By this, a coefficient matrix $\boldsymbol{\alpha}^{*}$ of dimension (KS $\times N$ ) is obtained, containing the contributions of each MNP relaxation type rowwise. In the second subproblem, an inverse problem $\mathbf{X}_{n}^{*}=\operatorname{argmin}\left\|\left(\mathbf{L}\left(X_{n}, \kappa_{n}\left(t_{1}, t_{2}\right)\right) \mathbf{X}_{n}\right)-\boldsymbol{\alpha}_{n}^{*}\right\|$ is solved independently for each MNP relaxation type and the result $\mathbf{X}_{n}^{*}$ assigned to a different color. Finally, an overlay of all $\mathbf{X}_{n}^{*}$ is created to generate the multicolor MRX image. 
The measured relaxation in the experiment $B_{\text {meas }}(t)$ is then modeled as a linear combination of the $N$ reference relaxations $\alpha_{n} \kappa_{n}(t)$. Consequently, the first subproblem can be solved by estimating the coefficients $\boldsymbol{\alpha}^{*}$ in $[T]$

$$
\boldsymbol{\alpha}^{*}=\underset{\alpha}{\operatorname{argmin}}\left\|\left(\sum_{N} \alpha_{n} \kappa_{n}(t)\right)-B_{\text {meas }}(t)\right\|
$$

to split-up $B_{\text {meas }}(t)$ into the contributions of the different phases. This problem is solved using NNLS (Equation 9.7) assuming the absolute contributions to be positive values, which is a correct approximation after signal filtering. Thus, $\boldsymbol{\alpha}^{*}$ (dimension $1 \times N$ ) contains the relative contributions of the $N$ phases in a single MRX measurement. Applying Equation (9.14) to the complete MRXI data set, i.e. $K$ MRX measurements of $S$ sensors, yields the final coefficient matrix $\boldsymbol{\alpha}^{*}$ of dimension $(K S \times N)$.

The solution of this first subproblem in the example in Figure 9.8 separates the signal into $N=4$ phases originating from MNPs in suspension, in blood, in cells and immobilized in a tissue matrix, respectively. Therefore, we first prepare MNP reference samples of known MNP mass $X_{\text {ref }}$ that emulate best the respective phase. Two reference MNP samples are simply suspended MNPs and immobilized MNPs, e.g., freeze-dried in a sugar matrix. The other two samples are prepared by diluting the MNP suspension in whole blood and the cell type of interest. These (small) samples are then consecutively measured by MRX to parameterize our data model (Equation 9.13) with $\chi_{n} \kappa_{n}(t)$. The four rows of the coefficient matrix $\boldsymbol{\alpha}^{*}$ obtained by Equation (9.14) contain the signal contributions of each phase for all MRX measurements of the MRXI sequence. Note that the signal separation can be advanced by the choice of appropriate time windows to evaluate each $\kappa_{n}(t)$ based on the reference properties (e.g., using only time frames when the signal-to-noise ratio of $\kappa_{n}(t)$ is above a certain limit) [59].

With this coefficient matrix, we enter the second subproblem which is the imaging problem. Here, Equation (9.7) is solved independently for each phase:

$$
\mathbf{X}_{n}^{*}=\underset{\mathbf{X}_{n}}{\operatorname{argmin}}\left\|\left(\mathbf{L}\left(\chi_{n}, \kappa_{n}\left(t_{1}, t_{2}\right)\right) \mathbf{X}_{n}\right)-\boldsymbol{\alpha}_{n}^{*}\right\|, \quad n=1, \ldots, N
$$

Since the time-dependence $\kappa(t)$ was already employed in the first subproblem for signal separation, it is sufficient to use the amplitude difference of $\kappa_{n}\left(t_{1}, t_{2}\right)$ in the imaging problem. As a result, we obtain independent 3D MRXI reconstructions $\mathbf{X}_{n}^{*}$ for each phase $n$. The overlay of all $N$ fractional MNP distributions $\mathbf{X}_{n}^{*}$ is the reconstructed MNP distribution obtained by multicolor MRXI. In our example in Figure 9.8, the second subproblem is solved independently for each row of $\boldsymbol{\alpha}^{*}=\left[\boldsymbol{\alpha}_{1}^{*}, \boldsymbol{\alpha}_{2}^{*}, \boldsymbol{\alpha}_{3}^{*}, \boldsymbol{\alpha}_{4}^{*}\right]$ to obtain four individual quantitative MNP reconstructions that belong to each phase of the MNP distribution. The final multicolor MRXI image is then the overlay of the four MNP distributions encoded by color to show the phase.

In Figure 9.9, we experimentally demonstrate multicolor MRXI on a MNP distribution measured in the ROI tumor of the rabbit-sized phantom [59]. The MNP distribution was assembled of MNPs in $N=4$ phases as listed in Table 9.3, while Figure 9.9 shows a 
photograph of the MNP support (a), the nominal MNP distribution (b), the resulting four fractional MRXI reconstructions (c) and their overlay (d). Note that except for the freeze-dried sample of F200 in which the particles were embedded in a solid phase, all MNPs were suspended in a liquid in our experiment. Hence, we mainly varied the MNP size distributions to generate distinct phases to emulate different PMIs. These systems allow us to investigate multicolor MRXI under well-defined experimental conditions. The MNP systems applied are fluid MAG-D (chemicell GmbH, Germany) and nanoMAG-D (micromod Partikeltechnologie GmbH, Germany).

The MNPs in the four phases are only placed one voxel $(1.2 \mathrm{~cm})$ from each other. In this case, the complexity of both multicolor MRXI subproblems increases compared to the previous MNP distribution (Figure 9.7) in which only MNPs in two spatially well-

a

MNP support
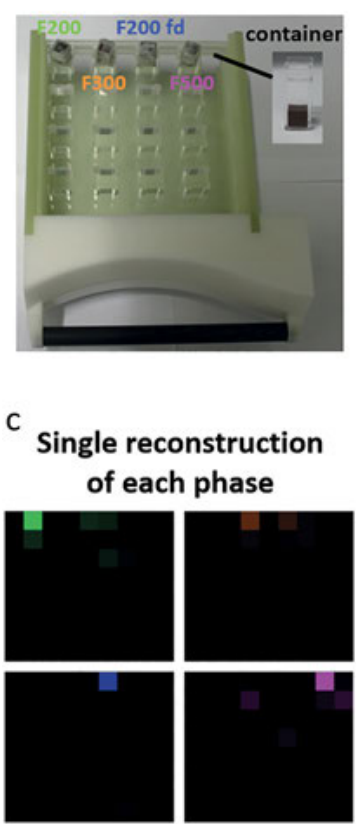

b
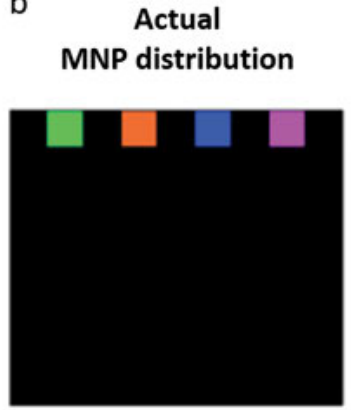

d

Multi-color MRXI reconstruction

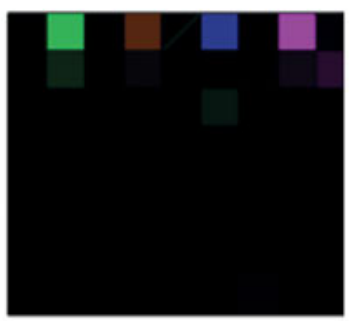

Particle sizes and amount

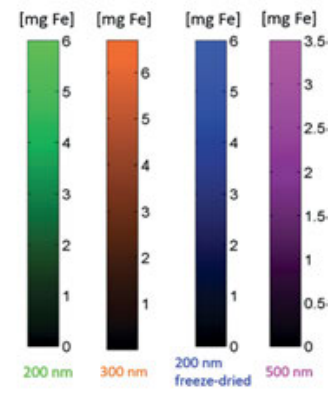

Figure 9.9: Multicolor MRX imaging of the MNP distribution assembled in the MNP support ROI tumor in the rabbit-sized phantom. (a) Photograph of the MNP support showing the spatial arrangement of MNPs in four different phases within their respective sample container $(V=0.5 \mathrm{~mL})$. (b) The nominal MNP distribution for the four MNPs phases with $200 \mathrm{~nm}$ MNPs (F200: green), $300 \mathrm{~nm}$ MNPs (F300: orange), $200 \mathrm{~nm}$ freeze-dried MNPs (F200 fd: blue) and $500 \mathrm{~nm}$ MNPs (F500: violet). Each sample fills a single voxel in the reconstruction grid of $10 \times 10$ voxel with a spatial resolution of $1.2 \mathrm{~cm}$, respectively. The four single-color reconstructions $\mathbf{X}_{n}^{*}$ are shown in (c) and their overlay is shown in (d). A high degree of similarity to the nominal MNP distribution is visible. Adapted with permission from Coene et al. [57]. Copyright 2017 IOP Publishing. 
Table 9.3: MNP properties under study.

\begin{tabular}{llrr}
\hline Name & Type & Size $(\mathbf{n m})$ & Amount $(\mathrm{mg}$ Fe) \\
\hline F200 & FluidMAG-D & 200 & 6 \\
F200 freeze-dried & FluidMAG-D & 200 & 6 \\
F300 & FluidMAG-D & 300 & 6.7 \\
N500 & NanoMAG-D & 500 & 3.5 \\
\hline
\end{tabular}

separated phases are present. The multicolor MRXI reconstructions, however, show excellent correspondence to the nominal MNP distribution. MNPs in the four phases are spatially well separated in the image that shows a correlation above $87 \%$ for all MNP distributions $\mathbf{X}_{n}^{*}$ and even $96 \%$ for F200. Even in imaging this four-phase MNP system, multicolor MRXI accomplishes a quantitative reconstruction with a total mass deviation below 30\%.

This clearly demonstrates the ability of multicolor MRXI to quantitatively image PMIs. MNP distributions containing MNPs in up to four different phases were quantitatively resolved with a sensitivity in the milligram/cubic centimeter range.

\subsection{Flow induced particle-matrix interactions}

\subsubsection{Microscale visualization of particle-matrix interactions in flow}

In this section, we investigate interactions between particles and flowing media. By magnetic monitoring of these interactions, we aim to employ the particles as local flow probes. On the one hand, this is important for biomedical MNP applications, where particle interactions with the circulatory system influence the efficiency of biomedical applications. On the other hand, this might enable the detection of perturbations in the hemodynamics, as, e.g., attributed to pathologic narrowing of vessels (atherosclerosis).

Inside the circulatory system, MNPs experience Stokes friction and shear forces generated by the blood flow. These forces result in translational and rotational MNP motion. In medical imaging (e.g., [63, 64]), translational MNP motion is visualized by mapping the transit of a contrast agent MNP bolus moving with the blood stream. However, the dispersion of MNPs in blood hardens blood flow quantification based on this motion. At the same time, rotational MNP motion that might give additional information on the hemodynamics is not visible.

Here, we detail a novel method called magnetic microsphere tracking (MMT) [65]. It might overcome these limitations by magnetic monitoring of translational and rotational particle motion. A single permanent magnetic microsphere (MM) with a diameter 
of a few micrometer locally probes the flow while passing through a vessel. The 304 SQUID sensor system [60] detects these movements and allows for high-resolution (millisecond/millimeter) 3D tracking of the translational and rotational motion of the sphere's magnetic moment in a field-of-view (FOV) of $15 \times 20 \mathrm{~cm}$. By this, we successfully localize and evaluate diameter constrictions in an arteria phantom. The size of the applied MM $(d=34.6 \mu \mathrm{m})$ is close to that of red blood cells $(6-8 \mu \mathrm{m})$ and demonstrates the potential for biomedical MMT application for blood flow quantification.

The concept of MMT is sketched in Figure 9.10. Here, a MM with permanent magnetic moment moves inside a vessel due to the hydrodynamic forces applied by the flow profile. The diameter of the vessel decreases along the $x$-coordinate due to increasing grades of stenosis. Thus, the flow vectors of the quadratic flow profile increase along $x$. A shear force acts on the MM due to variations of the flow vectors over the cross-section of the sphere. This force induces a rotational motion to the MM while drag forces translate it. Hence, the angle $\delta$ between MM magnetic moment and $x$-axis oscillates along $x$ at a certain frequency determined by the shear force. Thus, the rotational frequency of these oscillations increases with narrowing vessel diameter due to increasing shear forces. At the same time, the motions of the MM magnetic moment are remotely detected by a SQUID sensor at location $\mathbf{r}_{s}$ above the vessel. The amplitude of the SQUID signal depends on the actual position of the MM $\mathbf{r}_{v}(t)=\left[r_{x}(t), r_{y}(t), r_{z}(t)\right]$ relative to the SQUID. The distance dependency

$$
B_{s}(t)=\frac{\mu_{0}}{4 \pi}\left(\frac{3\left(\mathbf{n}^{\mathrm{T}}\left(\mathbf{r}_{s}-\mathbf{r}_{v}(t)\right)\left(\mathbf{r}_{s}-\mathbf{r}_{v}(t)\right)^{\mathrm{T}}\right)}{\left\|\left(\mathbf{r}_{s}-\mathbf{r}_{v}(t)\right)\right\|^{5}}-\frac{\mathbf{n}_{s}^{\mathrm{T}}}{\left\|\left(\mathbf{r}_{s}-\mathbf{r}_{v}(t)\right)\right\|^{3}}\right) \mathbf{m}(t)
$$

is employed to localize the actual position $\mathbf{r}_{v}(t)$ and magnetic moment $\mathbf{m}(t)=\left[m_{\mathbf{x}}(t)\right.$, $\left.m_{y}(t), m_{z}(t)\right]$ of the MM by the SQUID array iteratively for each discrete measured sample $t=\left[T_{1}, \ldots, T_{\text {end }}\right]$. This can be accomplished by solving the inverse problem

$$
\Omega\left(\mathbf{r}_{v}(t), \mathbf{m}(t)\right)=\underset{\Omega}{\operatorname{argmin}}\left\|\left[B_{1}(t), \ldots, B_{S}(t)\right]^{\mathrm{T}}-\mathbf{B}_{\text {meas }}(t)\right\|, \quad t=T_{1}, \ldots, T_{\text {end }} .
$$

using a Levenberg-Marquart optimizer $[66,67]$ to iteratively minimize the functional $\Omega\left(\mathbf{r}_{v}(t), \mathbf{m}(t)\right)$ by parameter variation $\mathbf{r}_{v}(t)$ and $\mathbf{m}(t)$. The inverse problem was simplified by the constant MM magnetic moment of $|\boldsymbol{m}|=10 \mathrm{nAm}^{2}$ that allows to reduce the model parameters to three MM coordinates in 3D space and the two angles $\alpha$ and $\beta$ defining the orientation of its magnetic moment, i.e. five degrees of freedom. This dipole localization was applied for each measured sample recorded during the MM passage through a flow phantom with a sampling frequency of $750 \mathrm{~Hz}$, i.e. a temporal resolution of round $1.3 \mathrm{~ms}$. We calculated the translational MM movement as first derivate of $\mathbf{r}_{v}^{\prime}(t)$ over time and estimated the MM rotational frequency by the oscillations of the angles $\alpha$ and $\beta$. For further details see [65]. 


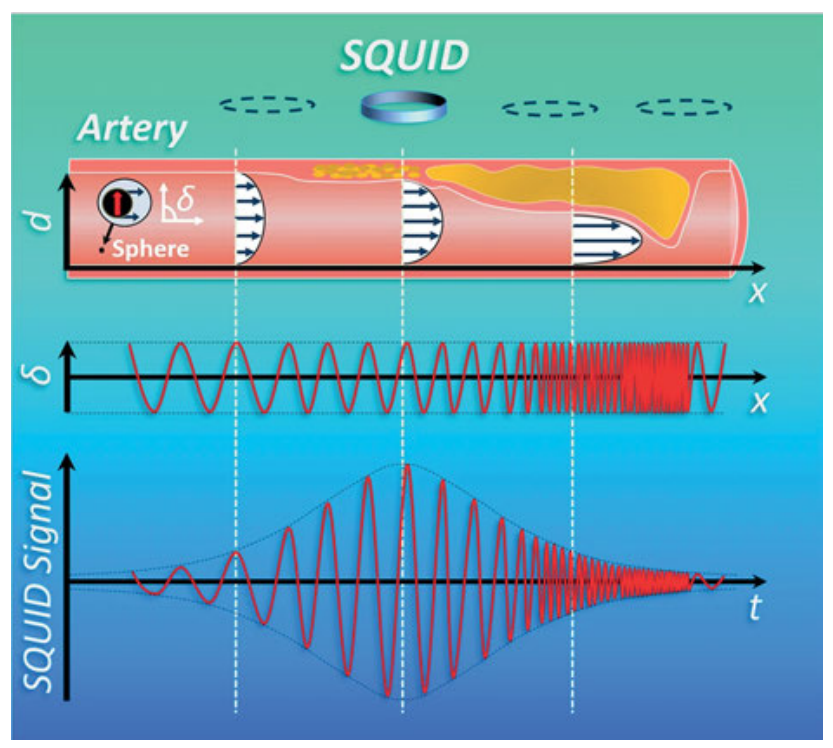

Figure 9.10: Principle of magnetic microsphere tracking: a microsphere with permanent magnetic moment passes an artery with increasing diameter constrictions (grades of stenosis) and thus, increasing velocity vectors of the quadratic flow profile. Due to the shear forces, the angle delta between magnetic moment and $x$-axis will oscillate along $x$ with increasing frequency. This oscillation is then detected by a SQUID and the actual sphere position tracked by a sensor array. Reproduced from Ref. [63] under the Creative Commons Attribution 4.0 International License.

\subsubsection{Tracking a single magnetic microsphere to monitor the fluid dynamics}

Here, we apply MMT to visualize PMIs in the flow phantom depicted in Figure 9.11a [65]. It consists of a straight tube section with a constriction region of $1 \mathrm{~cm}$ length. Here, variable diameter reductions $\mathrm{dr}$ of the original tube diameter $\left(d_{\text {tube }}=1.5 \mathrm{~mm}\right)$ of up to $50 \%$ can be adjusted. An additional curved tube section provides a secondary flow (Dean effect [68]) resulting in lateral migrations of the MM whether toward the tube center or wall. Note that these migrations would either lead to an increase in velocity and decrease in rotational frequency when the MM migrates toward the centerline or vice-versa. A constriction, however, results in a temporary increase in both, MM velocity and rotational frequency. The MM was separated from commercial Magnequench $^{\circledR}$ powder (MQP'T $\left.-S-11-9\right)$ using a cell selector $\left(\right.$ ALS $^{\circledR}$ ) and magnetized at a flux density of $5 \mathrm{~T}$. The flow media was pumped by a syringe pump (Landgraf ${ }^{\circledR}$, LA800) at a constant flow rate of $30 \mathrm{~mL} / \mathrm{min}(\operatorname{Re}=212)$.

The localized $x$ - and $y$-positions obtained by MMT are depicted in Figure 9.11b. The path of the MM is encoded by color to show the rotational MM frequency $f_{\text {rot }}$. For an 
a

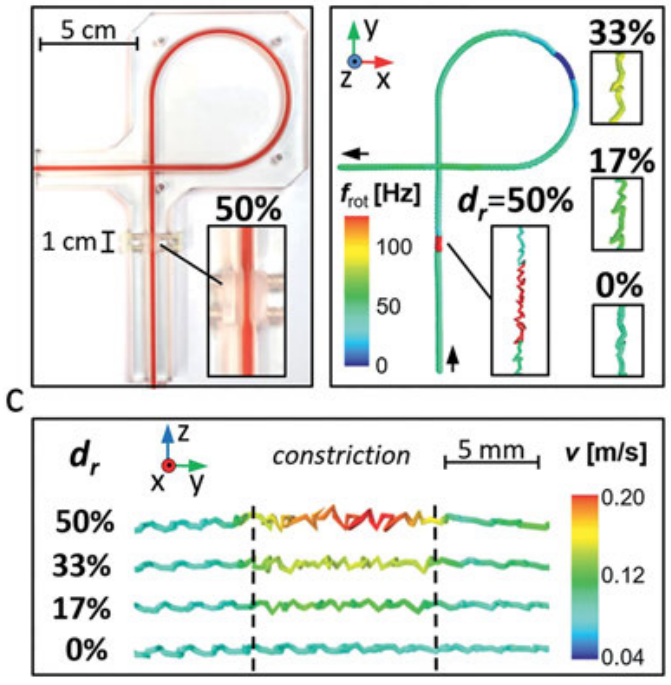

Figure 9.11: Magnetic Microsphere Tracking in a flow phantom. (A) The flow phantom comprising a straight and a curved tube section. In the straight section variable constrictions can be applied over a length of $1 \mathrm{~cm}$ using four nylon screws. (B) Localized $x, y$-position of the MM color coded to show the rotational MM frequency. The insets show an enlargement of the constriction region. A systematically increasing rotational MM frequency with increasing diameter reduction $d_{\mathrm{r}}$ is visible. (C) Enlargement of the localized $y, z$ - positions of the MM in the constriction region for different diameter reductions. The MM trace is color coded to show the MM velocity. A clear acceleration is seen when the MM enters the constriction and it decelerates when it leaves the constriction. Reproduced from Ref. [63] under the Creative Commons Attribution 4.0 International License.

adjusted diameter constriction of $d_{\mathrm{r}}=50 \%$, a clear increase of the rotational frequency is visible when the MM passes the constriction region and it remains constant in the absence of a constriction. The enlargements in Figure 9.11b show MM path and rotational frequency through the constriction region for variable diameter constrictions. A decrease of the rotational frequency is observed with increasing cross-section of the tube.

As expected, a decrease of the rotational frequency is also visible in the curved tube section when the MM migrates toward the tube center due to the applied secondary flow.

Figure 9.11c shows an enlarged view of the localized MM positions ( $y-z$ plane) during the passage through the constriction region for different diameter reductions. The color of the MM path is encoded by color to show the local velocity $v$. An abrupt acceleration is visible when the MM enters the constriction, and it decelerates again when it has left the constriction. Inside the constriction region, the MM velocity 
increase systematically with decreasing tube diameter, while it remains unchanged in the absence of a constriction. Hence, the degree of constriction has a clear impact on the MMT localization parameters frequency and velocity.

Hence, MMT allows to visualize microscale PMIs in flow by tracking a single microsphere (size range of a red blood cell) with a temporal resolution of $1.3 \mathrm{~ms}$ and a spatial resolution below $1 \mathrm{~mm}$. From the measurements we were able to quantify the flow conditions experienced by the microsphere. This enables localization and quantification of constricted volumes and distinction from lateral MM migrations. Additionally, separating influences of Stokes friction and shear flow makes MMT an ideal tool for rheology investigations and might help to understand complex flow dynamics. By its properties (quantitative, high resolution, no radiation, no tissue contributions, low marker dose [ng]) MMT holds great potential for biomedical blood flow quantification (more details in [65]).

\subsubsection{Sensing of particle-matrix interactions in flow on the nanoscale}

In the following, we investigate flow-induced PMIs in the nanoscale using MRX [69]. To accomplish this, we combine our MRX device comprising of a SQUID detector and a magnetizing coil with a flow phantom as sketched in Figure 9.12a. For flow experiments, $3 \mathrm{~mL}$ of a homogeneous MNP solution was injected into a $5 \mathrm{~m}$ long PVC tube with inner diameter of $0.8 \mathrm{~mm}$. Inside the magnetizing coil of the MRX device, i.e. the sensing volume, the tube was arranged to form a coil with five turns. The field vectors of the applied magnetic field were oriented perpendicular to the flow direction. The MNP
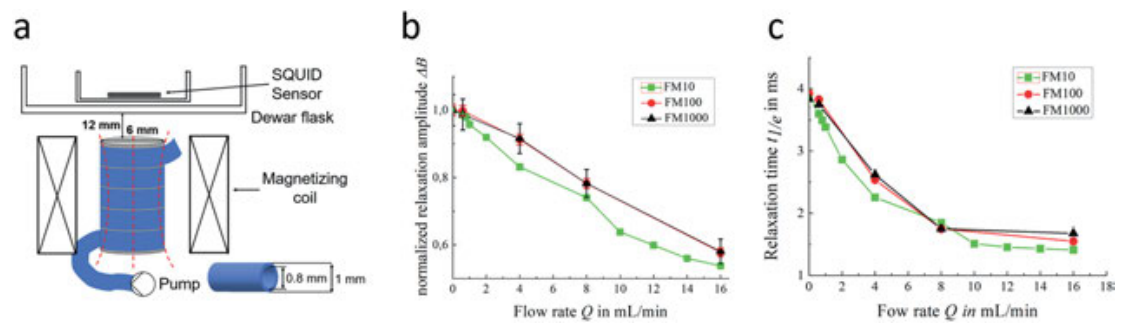

Figure 9.12: MRX measurements in flow. (a) Scheme of the experimental setup for the MRX measurements of MNPs under flow conditions. Driven by a syringe pump, the MNPs are flowing through the tube inside the MRX magnetizing coil of the MRX measurement device. To increase the sample volume inside the magnetizing coil, the tube is coiled up on a nonmagnetic core with five turns. The magnetizing coil is placed under the bottom of the SQUID sensor for detection of the magnetic relaxation. On the right, relaxation amplitudes $\Delta B(\mathrm{~b})$ and relaxation times $t_{1 / e}$ (c) as function of the flow rate $Q$ are depicted. These were obtained for dilutions of the original MNP suspension by a ratio of 1:10 (FM10), 1:100 (FM100), 1:1000 (FM1000) with BSA. Adapted with permission from Slabu et al. [67]. Copyright 2017 IOP Publishing. 
suspension was pumped through the tubing system by a syringe pump (LA-120, New Era Pump Systems, USA) at a constant flow rate. MRX measurements were performed with constant flow rates adjusted between 0.6 up to $16 \mathrm{~mL} / \mathrm{min}$. We used homogeneous fluidMAG/12-HS/130 (chemicell GmbH, Germany) suspensions of iron concentration $c=(2.8,0.28,0.028 \mathrm{mg} / \mathrm{mL})$, respectively. In the MRX magnetizing phase, an external magnetic field of $2 \mathrm{mT}$ was applied to the MNP suspension for magnetizing time of $1 \mathrm{~s}$. In the measurement phase, the resulting MRX signal was recorded by the SQUID sensor for a measurement time of $0.5 \mathrm{~s}$ at a sampling frequency of $100 \mathrm{kHz}$.

The characteristic MRX parameters relaxation amplitude $\Delta B$ and relaxation time $t_{1 / e}$ are depicted in Figure 9.12b and c as a function of the flow rate $Q$, respectively. Independent on MNP concentration, a clear decrease in both parameters with increasing flow rate is visible. Hence, shear-stress induced rotational MNP motion fastens Brownian relaxation and decreases the relaxation amplitude. Interestingly, while $\Delta B$ decreases proportional to the applied flow rate, the decrease of $t_{1 / e}$ nearly saturates for flow rates above $8 \mathrm{~mL} / \mathrm{min}$. This effect is reproducibly visible in all three MNP concentrations. This saturation behavior indicates a change in the tube flow profile. Slight concentration dependent variations, i.e. faster decreasing relaxation amplitudes and relaxation times for the higher concentrated FM10 are visible as well. This might be due to MNP provoked shear thinning and structure formation as, e.g., nanoparticle chain formations [69].

To summarize, we sensitively detect flow-induced changes in the Brownian relaxation of MNPs by MRX measurements. By analyzing the characteristic MRX parameters relaxation amplitude and relaxation time, we apply the MNPs as local probes to quantify flow and determine perturbations in the flow profile.

\subsection{Summary and conclusion}

We reviewed magnetic measurement methods that sensitively detect particle-matrix interactions and thereby, access the physical properties of the molecular MNP environment. For selected MNP-matrix systems, we demonstrated that the combination of different noninvasive and sensitive magnetic measurement techniques for specific MNP detection is a powerful approach to provide insights into dynamic processes of the MNPs as, e.g., binding kinetics, cellular uptake, passage through cell barriers and heat induction.

Furthermore, we developed functional imaging approaches that allow for spatially resolved investigation of particle-matrix interactions. Here, we utilized the impact of particle-matrix interactions on the MNP relaxation to advance MRX imaging with a multicolor approach and to resolve the location, quantity and molecular environment of MNPs. This technique might become a valuable tool to visualize and quantify particle-matrix interactions in preclinical research and propelling the development of future particle mediated therapies. 
As MNPs in nearly all biomedical applications are subject to body fluids and their prevailing fluid dynamics, we investigated flow-induced particle interactions. These can be detected by sensitive magnetic measurement methods to quantify the fluid dynamics acting on particles in the micro- and nanoscale. Magnetic microsphere tracking allows for the spatially resolved quantification of the fluid dynamics based on the particle velocity and rotational frequency with excellent temporal and spatial resolution. This novel method has great potential to become a valuable tool for rheology or even biomedical blood flow quantification and to pave the way for a deeper understanding of flow-induced particle dynamics inside the body.

Though countless MNP systems with sizes in the nanometer and micrometer range have been developed and applied for biomedical applications in recent years, there still is strong demand for further MNP research. To facilitate this, an accompanying development and improvement of established measurement techniques and analysis methods for MNPs and their interaction with biological environment is a mandatory prerequisite.

Author contributions: All the authors have accepted responsibility for the entire content of this submitted manuscript and approved submission.

Research funding: The authors gratefully acknowledge financial support by the DFG priority program SPP1681 (grant nos. WI 4230/1-3, MA 2432/6-2, KR 3929/2-1/2). A. C. and J. L. are supported by the Research Foundation - Flanders (FWO) through a postdoctoral fellowship. AC and JL were guest scientists at PTB with the support of PTB and the FWO (V409915N, AC and V410015N, JL).

Conflict of interest statement: The authors declare no conflicts of interest regarding this article.

\section{References}

1. Gupta AK, Gupta M. Synthesis and surface engineering of iron oxide nanoparticles for biomedical applications. Biomaterials 2005;26:3995-4021.

2. Hergt R, Dutz S. Magnetic particle hyperthermia-biophysical limitations of a visionary tumour therapy. J Magn Magn Mater 2007;311:187-92.

3. Pankhurst QA, Connolly J, Jones SK, Dobson J. Applications of magnetic nanoparticles in biomedicine. J Phys D Appl Phys 2003;36:R167.

4. Dutz S, Hergt R. Magnetic particle hyperthermia - a promising tumour therapy? Nanotechnology 2014;25:452001.

5. Poller JM, Zaloga J, Schreiber E, Unterweger H, Janko C, Radon P, et al. Selection of potential iron oxide nanoparticles for breast cancer treatment based on in vitro cytotoxicity and cellular uptake. Int J Nanomed 2017;12:3207.

6. Lugert S, Unterweger H, Mühlberger M, Janko C, Draack S, Ludwig F, et al. Cellular effects of paclitaxel-loaded iron oxide nanoparticles on breast cancer using different $2 \mathrm{D}$ and $3 \mathrm{D}$ cell culture models. Int J Nanomed 2019;14:161. 
7. Slabu I, Roeth AA, Engelmann UM, Wiekhorst F, Buhl EM, Neumann UP, et al. Modeling of magnetoliposome uptake in human pancreatic tumor cells in vitro. Nanotechnology 2019;30: 184004.

8. Ottersbach A, Mykhaylyk O, Heidsieck A, Eberbeck D, Rieck S, Zimmermann K, et al. Improved heart repair upon myocardial infarction: combination of magnetic nanoparticles and tailored magnets strongly increases engraftment of myocytes. Biomaterials 2018;155:176-90.

9. Vosen S, Rieck S, Heidsieck A, Mykhaylyk O, Zimmermann K, Bloch W, et al. Vascular repair by circumferential cell therapy using magnetic nanoparticles and tailored magnets. ACS Nano 2016; 10:369-76.

10. Poller WC, Pieber M, Boehm-Sturm P, Ramberger E, Karampelas V, Möller K, et al. Very small superparamagnetic iron oxide nanoparticles: long-term fate and metabolic processing in atherosclerotic mice. Nanomed Nanotechnol Biol Med 2018;14:2575-86.

11. Engelmann UM, Roeth AA, Eberbeck D, Buhl EM, Neumann UP, Schmitz-Rode T, et al. Combining bulk temperature and nanoheating enables advanced magnetic fluid hyperthermia efficacy on pancreatic tumor cells. Sci Rep 2018;8:13210.

12. Wells J, Löwa N, Paysen H, Steinhoff U, Wiekhorst F. Probing particle-matrix interactions during magnetic particle spectroscopy. J Magn Magn Mater 2019;475:421-8.

13. Weidner A, Gräfe C, von der Lühe M, Remmer H, Clement JH, Eberbeck D, et al. Preparation of coreshell hybrid materials by producing a protein corona around magnetic nanoparticles. Nanoscale Res Lett 2015;10:282.

14. Dutz S, Weidner A, von der Lühe M, Gräfe C, Biehl P, Demut J, et al. Hybrid nanomaterials of biomolecule corona coated magnetic nanoparticles and their interaction with biological systems. Phys Sci Rev 2020. https://doi.org/10.1515/psr-2019-0110. In press.

15. Deuflhard M, Eberbeck D, Hietschold P, Wilharm N, Mühlberger M, Friedrich RP, et al. Magnetically responsive composites: electron beam assisted magnetic nanoparticle arrest in gelatin hydrogels for bioactuation. Phys Chem Chem Phys 2019;21:14654-62.

16. Gräfe C, Müller EK, Gresing L, Weidner A, Radon P, Friedrich RP, et al. Magnetic hybrid materials interact with biological matrices. Phys Sci Rev 2020. https://doi.org/10.1515/psr-2019-0114. In press.

17. Gräfe C, Slabu I, Wiekhorst F, Bergemann C, Von Eggeling F, Hochhaus A, et al. Magnetic particle spectroscopy allows precise quantification of nanoparticles after passage through human brain microvascular endothelial cells. Phys Med Biol 2016;61:3986.

18. Heid S, Unterweger H, Tietze R, Friedrich RP, Weigel B, Cicha I, et al. Synthesis and characterization of tissue plasminogen activator-functionalized superparamagnetic iron oxide nanoparticles for targeted fibrin clot dissolution. Int J Mol Sci 2017;18:1837.

19. Müller EK, Gräfe C, Wiekhorst F, Bergemann C, Weidner A, Dutz S, et al. Magnetic nanoparticles interact and pass an in vitro co-culture blood-placenta barrier model. Nanomaterials 2018;8:108.

20. Nèel L. Théorie du traînage magnétique des ferromagnétiques en grains fins avec application aux terres cuites. Ann Geophys 1949;5:99-136.

21. Einstein A. Investigations on the theory of the Brownian movement. New York: Courier Corporation; 1956.

22. Kötitz R, Fannin PC, Trahms L. Time domain study of Brownian and Néel relaxation in ferrofluids. J Magn Magn Mater 1995;149:42-6.

23. Ludwig F, Heim E, Menzel D, Schilling M. Investigation of superparamagnetic $\mathrm{Fe}_{3} \mathrm{O}_{4}$ nanoparticles by fluxgate magnetorelaxometry for use in magnetic relaxation immunoassays. J Appl Phys 2006; 99:08P106.

24. Bessais L, Ben Jaffel L, Dormann JL. Relaxation time of fine magnetic particles in uniaxial symmetry. Phys Rev B 1992;45:7805. 
25. Liebl M, Steinhoff U, Wiekhorst F, Haueisen J, Trahms L. Quantitative imaging of magnetic nanoparticles by magnetorelaxometry with multiple excitation coils. Phys Med Biol 2014;59: 6607-20.

26. Baumgarten D, Liehr M, Wiekhorst F, Steinhoff U, Münster $P$, Miethe $P$, et al. Magnetic nanoparticle imaging by means of minimum norm estimates from remanence measurements. Med Biol Eng Comput 2008;46:1177.

27. Adolphi NL, Butler KS, Lovato DM, Tessier TE, Trujillo JE, Hathaway HJ, et al. Imaging of Her2targeted magnetic nanoparticles for breast cancer detection: comparison of SQUID-detected magnetic relaxometry and MRI. Contrast Media Mol Imaging 2012;7:308-19.

28. Steinhoff U, Wiekhorst F, Baumgarten D, Haueisen J, Trahms L. Bildgebung magnetischer nanopartikel basierend auf magnetrelaxometrie mit sequentieller aktivierung inhomogener anregungsfelder. Biomed Tech 2010;55:22-5.

29. Coene A, Crevecoeur G, Dupre L. Adaptive control of excitation coil arrays for targeted magnetic nanoparticle reconstruction using magnetorelaxometry. IEEE Trans Magn 2012;48:2842-5.

30. Liebl M, Steinhoff U, Wiekhorst F, Coene A, Haueisen J, Trahms L. Quantitative reconstruction of a magnetic nanoparticle distribution using a non-negativity constraint. Biomed Tech 2013;58(1): 000010151520134261.

31. Lawson CL, Hanson RJ. Linear least squares with linear inequality constraints solving least squares problems. Englewood Cliffs, NJ: Prentice-Hall; 1974:158-73 pp.

32. Debye PJW. Polar molecules. New York: Chemical Catalog Company, Incorporated; 1929.

33. Ficko BW, Nadar PM, Hoopes PJ, Diamond SG. Development of a magnetic nanoparticle susceptibility magnitude imaging array. Phys Med Biol 2014;59:1047.

34. Ficko BW, Nadar PM, Diamond SG. Spectroscopic AC susceptibility imaging (sASI) of magnetic nanoparticles. J Magn Magn Mater 2015;375:164-76.

35. Baffa O, Corá LA, Américo MF, Fonseca PR, Oliveira RB, Miranda JR. Magnetic images of pharmaceutical dosage forms in the human gastrointestinal tract. In: Annual international conference of the IEEE engineering in medicine and biology - proceedings; 2005.

36. Soares G, Próspero A, Calabresi M, Rodrigues D, Simoes L, Quini C, et al. Multichannel AC biosusceptometry system to map biodistribution and assess the pharmacokinetic profile of magnetic nanoparticles by imaging. IEEE Trans NanoBioscience 2019;18:456-62.

37. Gleich B, Weizenecker J. Tomographic imaging using the nonlinear response of magnetic particles. Nature 2005;435:1214.

38. Pessot G, Cremer P, Borin DY, Odenbach S, Löwen H, Menzel AM. Structural control of elastic moduli in ferrogels and the importance of non-affine deformations. J Chem Phys 2014;141:015005.

39. Deng H-X, Gong X-I, Wang L-H. Development of an adaptive tuned vibration absorber with magnetorheological elastomer. Smart Mater Struct 2006;15:N111.

40. Varga Z, Fehér J, Filipcsei G, Zrínyi M. Smart nanocomposite polymer gels. In: Macromolecular symposia; 2003.

41. Eberbeck D, Bergemann C, Wiekhorst F, Steinhoff U, Trahms L. Quantification of specific bindings of biomolecules by magnetorelaxometry. J Nanobiotechnol 2008;6:1-12.

42. Wisotzki El, Eberbeck D, Kratz H, Mayr SG. Magnetic response of gelatin ferrogels across the solgel transition: the influence of high energy crosslinking on thermal stability. Soft Matter 2016;12: 3908-18.

43. Kraupner A, Eberbeck D, Heinke D, Uebe R, Schüler D, Briel A. Bacterial magnetosomes - nature's powerful contribution to MPI tracer research. Nanoscale 2017;9:5788-93.

44. Eberbeck D, Wiekhorst F, Steinhoff U, Trahms L. Aggregation behaviour of magnetic nanoparticle suspensions investigated by magnetorelaxometry. J Phys Condens Matter 2006;18:S2829. 
45. Astalan AP, Ahrentorp F, Johansson C, Larsson K, Krozer A. Biomolecular reactions studied using changes in Brownian rotation dynamics of magnetic particles. Biosens Bioelectron 2004;19: 945-51.

46. Kammel M, Hoell A, Wiedenmann A. Structure of magnetite ferrofluids investigated by SANS with polarized neutrons. Scripta Mater 2001;44:2341-5.

47. Avdeev MV, Aksenov VL. Small-angle neutron scattering in structure research of magnetic fluids. Phys Usp 2011;53:971-93.

48. Nagornyi AV, Bulavin LA, Petrenko VI, Avdeev MV, Aksenov VL. Sensitivity of small-angle neutron scattering method at determining the structural parameters in magnetic fluids with low magnetite concentrations. Ukr J Phys 2013;58:735-41.

49. Narayanan $\mathrm{T}$, Wacklin $\mathrm{H}$, Konovalov $\mathrm{O}$, Lund R. Recent applications of synchrotron radiation and neutrons in the study of soft matter. Crystallogr Rev 2017;23:160-226.

50. Frielinghaus H, Feoktystov A, Berts I, Mangiapia G. KWS-1: small-angle scattering diffractometer. J Large-Scale Res Facil JLSRF 2015;1:28.

51. Feoktystov AV, Frielinghaus H, Di Z, Jaksch S, Pipich V, Appavou MS, et al. KWS-1 high-resolution small-angle neutron scattering instrument at JCNS: current state. J Appl Crystallogr 2015.

52. Pipich V. QtiKWS: user-friendly program for reduction, visualization, analysis and fit of SA(N)S data. Software Toolbox for SANS Data; 2012. http://www.qtikws.de [Accessed 30 Mar 2021].

53. Szczerba W, Costo R, Veintemillas-Verdaguer S, Del Puerto Morales M, Thünemann AF. SAXS analysis of single- and multi-core iron oxide magnetic nanoparticles. J Appl Crystallogr 2017;50: 481-8.

54. Zhang F, Skoda MW, Jacobs RM, Martin RA, Martin CM, Schreiber F. Protein interactions studied by SAXS: effect of ionic strength and protein concentration for BSA in aqueous solutions. J Phys Chem B 2007;111:251-9.

55. Winiewska M, Szewczuk-Karpisz K, Sternik D. Adsorption and thermal properties of the bovine serum albumin-silicon dioxide system. J Therm Anal Calorim 2015;120:1355-64.

56. Nosrati H, Salehiabar M, Fridoni M, Abdollahifar MA, Kheiri Manjili H, Davaran S, et al. New insight about biocompatibility and biodegradability of iron oxide magnetic nanoparticles: stereological and in vivo MRI monitor. Sci Rep 2019;9:1-10.

57. Muri HI, Hoang L, Hjelme DR. Mapping nanoparticles in hydrogels: a comparison of preparation methods for electron microscopy. Switzerland: Applied Sciences; 2018.

58. Liebl M, Wiekhorst F, Eberbeck D, Radon P, Gutkelch D, Baumgarten D, et al. Magnetorelaxometry procedures for quantitative imaging and characterization of magnetic nanoparticles in biomedical applications. Biomed Tech 2015;60:427-43.

59. Coene A, Leliaert J, LiebI M, Löwa N, Steinhoff U, Crevecoeur G, et al. Multi-color magnetic nanoparticle imaging using magnetorelaxometry. Phys Med Biol 2017;62:3139-57.

60. Schnabel A, Burghoff M, Hartwig S, Petsche F, Steinhoff U, Drung D, et al. A sensor configuration for a 304 SQUID vector magnetometer. Neurol Clin Neurophysiol Annu NCN 2004;2004:70.

61. Alexiou C, Arnold W, Klein RJ, Parak FG, Hulin P, Bergemann C, et al. Locoregional cancer treatment with magnetic drug targeting. Canc Res 2000;60:6641-8.

62. Wiekhorst F, Liebl M, Steinhoff U, Trahms L, Lyer S, Dürr S, et al. Magnetorelaxometry for in-vivo quantification of magnetic nanoparticle distributions after magnetic drug targeting in a rabbit carcinoma model. Springer Proc Phys 2012;140:301-5.

63. Nicol ED, Padley SPG. Non-invasive cardiac imaging: current and emerging roles for multi-detector row computed tomography. Part 2. Br J Cardiol 2007;14:237-41.

64. Gordon Y, Partovi S, Müller-Eschner M, Amarteifio E, Bäuerle T, Weber M-A, et al. Dynamic contrastenhanced magnetic resonance imaging: fundamentals and application to the evaluation of the peripheral perfusion. Cardiovasc Diagn Ther 2014;4:147-64. 
65. Liebl M, Gleich B, Eberbeck D, Radon P, Rahmer J, Trahms L, et al. Noninvasive monitoring of blood flow using a single magnetic microsphere. Sci Rep 2019;9:1-8.

66. Levenberg K. A method for the solution of certain non-linear problems in least squares. Q Appl Math 1944;2:164-8.

67. Marquardt DW. An algorithm for least-squares estimation of nonlinear parameters. J Soc Ind Appl Math 1963;11:431-41.

68. Dean WR. XVI. Note on the motion of fluid in a curved pipe. Lond Edinb Dublin Philos Mag J Sci 1927; 4:208-23.

69. Slabu I, Liebl M, Wiekhorst F, Eberbeck D. Magnetic relaxation of magnetic nanoparticles under the influence of shear flow. J Phys D: Appl Phys 2019;52:1-9. 
